\title{
Comparing Common Procedures Used to Manipulate Reinforcer Magnitude
}

Michael Steele Yencha

West Virginia University, msyencha@mix.wvu.edu

Follow this and additional works at: https://researchrepository.wvu.edu/etd

Part of the Applied Behavior Analysis Commons, and the Experimental Analysis of Behavior Commons

\section{Recommended Citation}

Yencha, Michael Steele, "Comparing Common Procedures Used to Manipulate Reinforcer Magnitude" (2021). Graduate Theses, Dissertations, and Problem Reports. 10324.

https://researchrepository.wvu.edu/etd/10324

This Dissertation is protected by copyright and/or related rights. It has been brought to you by the The Research Repository @ WVU with permission from the rights-holder(s). You are free to use this Dissertation in any way that is permitted by the copyright and related rights legislation that applies to your use. For other uses you must obtain permission from the rights-holder(s) directly, unless additional rights are indicated by a Creative Commons license in the record and/ or on the work itself. This Dissertation has been accepted for inclusion in WVU Graduate Theses, Dissertations, and Problem Reports collection by an authorized administrator of The Research Repository @ WVU.

For more information, please contact researchrepository@mail.wvu.edu. 
Comparing Common Procedures Used to Manipulate Reinforcer Magnitude

\author{
Michael Yencha
}

Dissertation Submitted to the Eberly College of Arts and Sciences at West Virginia University in Partial Fulfillment of the Requirements for the Degree of Doctor of Philosophy in Psychology.

\author{
Kennon A. Lattal, Ph.D., Chair \\ Barry Edelstein, Ph. D. \\ Genae Hall, Ph.D. \\ Kathryn Kestner, Ph.D. \\ Michael Perone, Ph. D. \\ Department of Psychology
}

Morgantown, West Virginia

2021

Keywords: Reinforcer Magnitude

Copyright 2021 Michael Yencha 


\begin{abstract}
Comparing Common Procedures Used to Manipulate Reinforcer Magnitude
\end{abstract}

Michael Yencha

Reinforcer magnitude is one of several parameters of reinforcement. In the present study, it referred to the quantitative value of the reinforcer in terms of duration of access to a finite supply of grain pellets. Pigeons responded on concurrent-chain schedules earning one of several reinforcer durations depending on response allocation and experimental conditions. Experiment 1 consisted of relatively long reinforcer durations available for completing one chain compared to relatively short durations available on the other. Response allocation in the initial link determined the reinforcer duration, but responses in the terminal link produced no change in the upcoming reinforcer. This arrangement allowed for the comparison of response-correlated and experimenter-controlled changes in reinforcer magnitude in terms of the control by reinforcer magnitude they produced. Additional changes in reinforcer durations were programmed between experimental conditions to allow for comparisons of within-sessions and between-conditions changes in magnitude as well. Experiment 2 was procedurally similar to the first, but the total reinforcer duration was identical for each chan. Instead, reinforcer durations were segmented into bins on one chain and kept continuous on the other (e.g., two 4-s reinforcers separated by a brief blackout period compared to one, continuous 8 -s reinforcer). Control by reinforcer magnitude was most apparent when changes were response correlated and within-sessions, but behavior change was also observed on the between-conditions scale. No magnitude effects were observed in changes in magnitude were experimenter controlled (i.e., not correlated to behavior). Further, the observed effects seemed to be mediated by eating efficiency. It is recommended that future research on reinforcer magnitude include thorough measurement of the consummatory chain to fully describe the role of eating efficiency in control by reinforcer magnitude. 


\section{Table of Contents}

Introduction.................................................................................1

Literature Review....................................................................5

Statement of the Problem................................................................17

Experiment 1............................................................................19

Method..................................................................................19

Results..................................................................................24

Discussion.......................................................................29

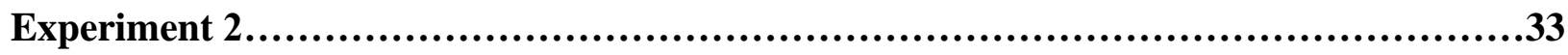

Introduction..........................................................................33

Method...............................................................................34

Results.................................................................................36

Discussion...........................................................................40

General Discussion..........................................................................44

References...............................................................................50

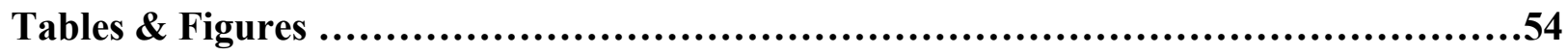





\section{Comparing Common Procedures Used to Manipulate Reinforcer Magnitude}

Parameters of reinforcement diminish or enhance reinforcer efficacy depending on their values. Contiguity between responding and reinforcement, for example, can be disrupted (thereby delaying reinforcer delivery from the response that produces it) to reduce systematically responding relative to when reinforcer delivery is immediate (Gleeson \& Lattal; 1987; Sizemore \& Lattal, 1978). As another case in point, choice (i.e., response allocation across multiple response options) tends to be directly related to the rate of reinforcement of each response (Fantino \& Davison, 1983; Herrnstein, 1970; McSweeney et al., 1995). That is, when two or more response options are available, responses most frequently are allocated to response options correlated with higher reinforcement rates. There is a long history of manipulating delay and frequency as reinforcement parameters that attests to the reliable behavior change they produce, lending credence to their description as basic principles of behavior analysis. Not all parameters of reinforcement, however, yield such consistent and reliable results when manipulated.

Reinforcer magnitude is the remaining parameter. Conceptually speaking, changes in reinforcer magnitude might be expected to produce systematic changes in behavior consistent with those resulting from manipulating other parameters (Bonem \& Crossman, 1988), but a large body of research on reinforcer magnitude describes various effects with differing degrees of systematicity and reliability. In a critical review of the reinforcement magnitude literature, Bonem and Crossman (1988) argued that these inconsistencies warrant additional analyses from the behavior-analytic community, suggesting that a systematic line of research be conducted to more clearly establish the conditions necessary and sufficient to observe systematic behavior change as a function of reinforcer magnitude. 
Some of the inconsistencies in reinforcement magnitude effects may be attributable to the different definitions of the term. That is, the reference is clear when an author describes manipulations of reinforcement rate or delay, but reinforcer magnitude is a parameter that can be manipulated across several dimensions. Some magnitude manipulations are quantitative, such as the concentration of a reinforcing solution, number of reinforcers presented per inter-reinforcer interval (IRI), and duration of access to a finite supply of a reinforcer (Bonem \& Crossman, 1988). Qualitative differences among reinforcers also have been conceptualized as variations in reinforcer magnitude. For example, preferences for different stimuli can be used to categorize reinforcers hierarchically as high magnitude (highly preferred) and low magnitude (lesser preferred) reinforcers (e.g., Ettinger et al., 1981). The question of reinforcer magnitude is complicated further in the case of reinforcer concentration, changes in which may be described quantitatively but necessarily involve a structural change in the reinforcer as well (e.g., comparing the taste of $50 \%$ sweetened milk and water solution to that of a solution of $10 \%$ sweetened milk and 90\% water). Additionally, changes in the various dimensionsldo not appear to be functionally equivalent (Bonem \& Crossman, 1988). That is, there is little evidence to suggest that a change in reinforcer duration, for example, would produce the same change in behavior that a change in the number of reinforcers (e.g., 1 vs 5 food pellets) would produce. Thus, generalizations about the effects of variations in the different dimensions of reinforcer magnitude must be made cautiously and with the support of empirical research. The different dimensions of reinforcer magnitude and their seeming functional inequivalence uniquely complicate the body of literature on reinforcer magnitude among reinforcement parameters, making it relatively difficult to identify systematicity within the findings and build on them in a systematic line of empirical evaluations. 
Additional factors that complicate the systematization of reinforcer magnitude effects are the many different procedures by which magnitude is commonly manipulated. These procedural variations include within-sessions, between-conditions, response-correlated, and experimentercontrolled changes in magnitude (Bonem \& Crossman, 1988). Within-session changes in magnitude are those which may occur in a single session, including arrangements of magnitude that vary from one response option to the next - a concurrent procedure - and those which vary magnitude in one component to the next, such as a mixed or multiple (mult) schedule. Betweenconditions changes in magnitude are those which occur after the behavior can be describes as stable according to visual inspection or some other criterion. Response-correlated changes in magnitude are those which are somehow contingent on the behavior of the subject. A concurrent schedule that provides different reinforcer magnitudes on each response option is an example of a response-correlated change in magnitude. Lastly, experimenter-controlled changes in reinforcer magnitude are those which occur independently of the subject's behavior and are instead arranged by the researcher. The use of a single schedule with reinforcer magnitude $\mathrm{X}$ in one condition compared to the same schedule with reinforcer magnitude $\mathrm{Y}$ in the next condition is an example of a response-independent change in reinforcer magnitude.

The major findings across the categories of procedures described above support the conclusion that within-session and response-correlated changes in magnitude produce the most systematic changes in behavior (Within session; Blakely \& Schlinger, 1988; Catania, 1963; Davison \& Baum; 2003; Dougherty \& Cherek, 1994; King \& Logue, 1990; Neuringer, 1967; Perone \& Courtney, 1992; Williams et al., 2011; Response correlated: Buskist, et al., 1988; Catania, 1963; Da Silva \& Lattal, 2010; Gentry \& Eskew, 1984; Keesey \& Kling, 1961; Landon et al., 2003; Neuringer, 1967), while their alternatives have produced mixed results ranging from 
inconsistent to systematic changes in behavior (Inconsistent; Catania, 1963; Neuringer, 1967; Systematic; Reed, 1991; Reed \& Wright, 1988). Bonem and Crossman (1988) stated that although these procedural variables may be manipulated to alter the effectiveness of reinforcer magnitude, none of these conditions have been identified as necessary to produce magnitude effects.

The progressive-ratio schedule is another common procedure in the reinforcer magnitude literature. It, unlike the other procedures described above which are used to maintain behavior in steady state, is used to evaluate behavior under strain. Using progressive ratios, experimenters like Baron et al. (1992) have evaluated relations between reinforcer magnitude and measures of behavioral persistence (i.e. breakpoint or the ratio value at which responding ceases for a predetermined period of time). Larger-magnitude reinforcers tend to produce relatively high breakpoints on progressive-ratio schedules when compared to smaller-magnitude reinforcers. That said, the current experiments were designed to capture magnitude effects in steady state behavior, and thus the literature review that follows is focused on those procedures described in Bonem and Crossman rather than the progressive-ratio schedule analyses of reinforcer magnitude effects.

The purpose of the current experiments was to compare some of the procedures that are commonly used to manipulate reinforcer magnitude and identify variables that are common among them. To that end, literature related to each of the common procedures used to manipulate reinforcer magnitude (excluding progressive ratios) is considered in the following review. The experiments deriving from this research literature were designed to evaluate changes in response rates that occur as a function of manipulating what are defined here as quantifiable dimensions of 
reinforcer magnitude. Thus, the review is limited to experiments that manipulated strictly quantitative dimensions of magnitude (viz., reinforcer duration and number of reinforcers.)

\section{Literature Review}

As described above, investigations of reinforcer magnitude (with the definition herein restricted to the number of reinforcers per IRI and/or duration of access to a reinforcer per IRI) can be categorized along two broad dimensions. First, whether those changes occurred within sessions (i.e., various magnitudes were available simultaneously in concurrent schedules or during the initial link of a concurrent chain, or alternated between components of multiple or mixed schedules) or between conditions (i.e., various magnitudes were presented across successive phases of an experiment). Second, whether the changes were correlated with response rate (i.e., different magnitudes were determined by choice or correlated with the number of responses in a given period) or occurred independently of subject behavior (i.e., magnitude was determined solely by the experimenter and changes occurred regardless of subject behavior). Because there are inconsistencies in the findings reported in each of those categories, the purpose of this review is to critique the methods and general findings of experiments in each of these categories and identify some of the controlling variables related to reinforcer magnitude effects shared among the categories.

\section{Response-Correlated and Experimenter-Controlled Changes in Reinforcer Magnitude}

Although no conditions have been identified as necessary to produce reinforcer magnitude effects, some have claimed that correlations between reinforcer magnitude and responding are sufficient to do so (Bonem \& Crossman, 1988). The positive results of several experiments that used response-correlated magnitude manipulations support that claim (Buskist

et al., 1988; Catania, 1963; Gentry \& Eskew; 1984; Neuringer, 1967). Neuringer (1967) directly 
compared response-correlated and response-independent changes in magnitude within individual subjects. Key pecking of pigeons was maintained with different durations of access to grain arranged by a concurrent-chain schedule. Initial links were comprised of concurrent fixed-ratio (FR) 1 FR 1 schedules. That is, the first response made to either of the keys was reinforced with the presentation of the terminal link on that key, and, simultaneously, the other key was deactivated for the remainder of the trial. Each terminal link consisted of a fixed-interval (FI) 5-s schedule on the active key. The first response after $5 \mathrm{~s}$ terminated the terminal link and lead to either $X$-s access to grain or a 1-s blackout. That is, terminal links ended in reinforcement only when a variable-interval (VI) schedule operating in the background of the experiment had elapsed, and blackouts occurred if the interval currently in effect had not yet lapsed. When a reinforcer became available, if the right key had been chosen in the initial link, then the reinforcer duration was always 2 s. Alternatively, reinforcer duration when the left initial-link key was chosen varied within a session in the following fixed order: $6,10,4,3,2.5,2$, and 2.25 s. All three pigeons responded on the left key more frequently than the right during the initial links, indicating that the correlation between choice responding and reinforcer duration was sufficient to control response allocation. Response rates in the terminal links, however, did not vary systematically as a function of the reinforcer magnitude, perhaps due to the lack of correlation between terminal link responding and reinforcer duration.

Neuringer's (1967) results, in isolation, do not allow the conclusion that responsecorrelated changes in magnitude are necessar to produce magnitude effects. Others who have used similar concurrent-chain schedules to compare response-correlated and experimentercontrolled changes in magnitude during initial and terminal links have reported mixed results. Specifically, both Schwartz (1969) and Lendenmann et al., (1982) reported that choice 
responding during initial links was a function of reinforcer duration, but, unlike Neuringer and Schwartz, Lendenmann et al. found systematic changes in terminal link response rates as a function of experimenter-controlled changes in reinforcer duration. The terminal links in Lendenmann et al. were relatively long (ranging from FI $30 \mathrm{~s}$ to VI $4 \mathrm{~min}$ ) compared to Neuringer's (1967) FI 5-s terminal links, allowing a relatively wide range of variability to occur which may account for the discrepant results. Like most areas within the reinforcer magnitude literature, the inconsistencies among the reported results suggest that certain controlling variables remain to be identified.

Another method of arranging response-correlated changes in magnitude is to increase the magnitude in a given IRI as a function of the number of responses occurring therein (Buskist, et al., 1988; Gentry \& Eskew, 1984). Gentry and Eskew (1984), for example, compared rates of pigeon key-peck responses when reinforcer duration varied based on the number of responses during an 8-s trial (response-correlated magnitude condition) or the duration remained fixed regardless of the number of responses during the trial (experimenter-controlled magnitude condition). The two conditions occurred on alternate days. In the response-correlated condition, each response in a trial added 0.25 -s access to grain to the 0.25 -s access that would occur at the end of the trial in the absence of responding. In the experimenter-controlled condition, a fixed 2.5-s period of access to food was delivered after each trial independently of the number of responses. Response rates were higher during the response-correlated condition than during the experimenter-controlled condition, indicating that responding was controlled by magnitude when reinforcer duration was correlated to response rate.

Gentry and Eskew (1984) also evaluated response rates during yoked-control conditions wherein the reinforcer duration at the end of each trial was correlated to the sequence of 
reinforcer durations that occurred each trial in the previous, response-correlated condition. One control condition required that at least one response occur before the reinforcer would be delivered, and the other ended after 8 s elapsed even if no responses had occurred. Response rates did not vary systematically as a function of the changes in magnitude that occurred within those yoked-control sessions, likely due to the lack of correlation between response rates and reinforcer duration. Buskist et al. (1988) conducted a similar evaluation of human operant behavior when reinforcer magnitude (number of points earned per IRI) was correlated with response rates for one group and varied independently of response rates for another. Response rates of the response-correlated group varied systematically as a function of magnitude, but the response rates of the experimenter-controlled group did not.

The experiments described thus far yielded positive relations between response rates and reinforcer magnitude when magnitude was correlated with choice or response rates; however, the use of response-correlated procedures is not strictly necessary to produce systematic magnitude effects. Others have found such effects as a function of experimenter-controlled changes in magnitude using mult schedules in which components with unequal amounts of reinforcement alternated (Blakely \& Schlinger, 1988; Perone \& Courtney, 1992; Williams et al., 2011). Blakely and Schlinger (1988), for example, maintained pigeon key-pecking responses with a mult VR $X$ VR $X$ schedule with 2-s reinforcer duration in one component and 8-s duration in the other. The values of $X$ were increased in each component during successive conditions. The postreinforcement pause (time between a reinforcer and the next response, hereafter labeled PRP) increased as a function of the increasing response requirement; however, PRPs in the 8-s reinforcer component increased by a relatively small amount compared to those in the 2-s reinforcer component. Similarly, Perone \& Courtney (1992) evaluated the key-pecking responses 
of pigeons maintained according to a mult FR FR schedule with equal ratio requirements in each component but varying reinforcer magnitudes across those components. One component produced a relatively short duration of access to grain once the ratio was completed and the other produced a relatively long duration of access to grain. The PRP increased in the component associated with the shorter reinforcer duration, but only when preceded by the component associated with the longer reinforcer duration (see also Williams et al., 2011). Those results, in combination with those of Blakely and Schlinger (1988), indicate that certain dimensions of behavior may be sensitive to within-sessions changes in reinforcer magnitude even when those changes are not correlated to response rates.

Although response-correlated changes in reinforcer magnitude appear to be sufficient to produce systematic behavioral changes given the broad findings reported in the literature, the role of within-session changes in reinforcer magnitude cannot be separated from the responsecorrelated results described thus far. Perhaps it would be more accurate to assert that the combination of within-sessions and response-correlated changes in magnitude appears to be sufficient to produce systematic magnitude effects.

\section{Within-Session and Between-Condition Changes in Reinforcer Magnitude}

Many of the experiments that produced positive relations between magnitude and behavior described in the above section necessarily involved within-session changes in magnitude due to the nature of response-correlated procedures. That is, when response allocation between concurrently available schedules or response rate criteria determined the magnitude of a given trial, those procedures could be described both as response-correlated and as withinsessions changes in magnitude. Thus, it is difficult to parse the relative effects of each type of procedure on behavior as a function of magnitude without comparing those results to the results 
of experiments that included yoked-control conditions, such as Gentry and Eskew (1984) and Buskist et al. (1988). Those reports showed that within-session changes in magnitude were insufficient to generate systematic behavior change in the absence of response-correlated changes in magnitude.

Although the majority of findings support the notion that within-sessions procedures more reliably produce systematic behavior change as a function of reinforcer magnitude than similar magnitude changes made between conditions (Blakely \& Schlinger, 1988; Lendenmann et al., 1982; Neuringer, 1967; Perone \& Courtney, 1992; Schwartz, 1969; Williams et al., 2011), in few experiments have the two procedures been compared directly. An exception is Catania (1963), which was an evaluation of the rate of pigeon key pecks maintained by different reinforcer durations when (a) reinforcer duration was manipulated between conditions (experimenter-controlled changes in magnitude) on single schedules and (b) reinforcer duration was varied within sessions and correlated with choice responding on concurrent schedules. Reinforcers in each condition were delivered according to VI schedules of reinforcement that produced either 3, 4.5, or 6-s access to food. Catania described the results as follows: with the single schedule procedure "[a] change in duration from 3.0 to $6.0 \mathrm{sec}$ had no systematic effect on the rate of key-pecking" and with the concurrent procedure "response rate was linearly related to reinforcement duration" (1963, p. 300). That is, if concurrently available response options produced either a relatively short or relatively long period of food access, then pigeons responded on the key that produced longer-access durations in each phase of the experiment. Alternatively, if there was no choice and the different reinforcer access durations were experimenter controlled, changing between conditions, then no systematic change in response rate occurred as a function of the access duration. 
Other investigators who have arranged different magnitudes of reinforcement within sessions using concurrent schedules have found similar results supporting the conclusion that reinforcer magnitude can systematically control choice behavior (Davison \& Baum, 2003; King \& Logue, 1990; Landon et al., 2003; Schmitt et al., 1974; Neuringer, 1967). Landon et al. (2003) used a switching-operandum concurrent VI VI procedure (Findley, 1958) to maintain pigeon key pecking. Responses to the left key (lit yellow) switched the color of the right key from red to green and vice versa. Responses to the right key produced access to grain in a hopper according to an interdependently scheduled, concurrent VI 30-s VI 30-s schedule. Under this concurrent schedule arrangement (cf. Stubbs \& Pliskoff, 1969), a reinforcer available for responses on one alternative had to be collected before more reinforcers would be arranged on the other alternative. Reinforcer magnitude was manipulated by delivering different numbers of 1.2-s food access periods during red and green alternatives. Each successive access period was separated by a .5-s blackout. The number of successive 1.2-s hopper presentations per IRI varied across red and green key light-color alternatives both within sessions (correlated to choice responses) and between conditions (arranged by experimenter). Conditions were arranged such that different ratios of reinforcer magnitude occurred across the red and green alternatives between conditions. Preference (response allocation and time spent in the presence of each key light) for the key light associated with the larger reinforcer magnitude occurred in each condition, and the preference was relatively greater during conditions where the discrepancy among red and green alternative magnitudes was relatively large compared to conditions where the magnitudes were closer to one another in value. The relatively high preference for the higher magnitude indicated that choice behavior was controlled by within-session changes in magnitude that were correlated to subject behavior, and the relative increase in said preference during conditions where the discrepancy 
between magnitudes was relatively large, compared to conditions where the magnitudes were more similar in value, indicated that choice also was sensitive to between-condition changes in magnitude.

Although Landon et al. (2003) reported systematic changes in choice as a function of both within-sessions and between-conditions changes in magnitude, Bonem and Crossman (1988) reported that relatively few experimenters using between-conditions changes in magnitude found systematic changes in behavior compared to those that used within-session changes in magnitude. Reed and Wright (1988) is one example of the former. They evaluated lever-pressing of rats maintained by a variable-ratio (VR) schedule. The VR value was the same throughout the experiment, but the number of pellets delivered per IRI varied across successive conditions. Running response rates (the number of responses divided by the IRI excluding the PRP, hereafter labeled Run Rates) increased as a function of increasing numbers of pellets for each rat. Additionally, PRP durations were relatively long in conditions with larger numbers of pellets. In a second experiment, a chained differential-reinforcement-of-low-rate (DRL) VR schedule-maintained lever pressing. In the initial, DRL link, the time between lever presses had to meet or exceed a certain time criterion to be reinforced with the presentation of the terminal, VR link. DRL and VR schedules typically generate very different patterns of responding, with the two schedules respectively maintaining relatively low and high response rates. When reinforcer magnitude was manipulated across conditions, DRL run rates (total number of responses in a session divided by session time minus total PRP) decreased (but see Doughty \& Richards, 2002, for a different effect) and those in VR increased. These findings suggest that control of responding by reinforcement magnitude does not always manifest as a rote change in response rates or PRP, but instead may manifest as increased schedule control. Thus, 
experimenters evaluating reinforcer magnitude effects should select dependent measures that are appropriate to capture behavior change in the context of the schedules of reinforcement in use.

Reed (1991) extended Reed and Wright's (1988) results using a mult VR VI schedule to compare between-conditions changes in magnitude across other schedules of reinforcement. In this experiment, VR and VI components alternated after a single reinforcer delivery followed by a 3-s blackout period, and each component produced an equal number of pellets in each condition. Reinforcer magnitude was manipulated across conditions that consisted of either 1 or 4 pellet deliveries per IRI. There was a 100-ms blackout between each of the 4 pellet deliveries earned per IRI during the 4-pellet condition. Response rates of two of the four rats increased in the VR component during the 4-pellet conditions relative to the 1-pellet condition, decreased for one rat, and showed no systematic change for the remaining rat. Response rates of three rats decreased in the VI component during the 4-pellet condition relative to the 1-pellet condition, and VI response rates of the remaining rat did not change systematically. These inconsistent findings support the claim that behavior maintained by different schedules of reinforcement may be differentially sensitive to reinforcer magnitude, but the lack of between-subjects replication suggests that controlling variables remain to be identified.

The findings of Reed and Wright (1988) and Reed (1991) show that between-conditions changes in magnitude can yield systematic magnitude effects, but the conditions necessary to observe those effects have not yet been identified. Other experimenters who have made similar manipulations, such as Catania (1963) have not reported systematic changes in behavior. One key difference in those experiments is the dimension of reinforcer magnitude manipulated therein. The former two experiments involved manipulations of the number of reinforcers per IRI, while the latter was a manipulation of reinforcer duration. 


\section{Functional Inequivalence of Reinforcer Duration and Number of Reinforcers}

Bonem and Crossman (1988) noted that the different dimensions of reinforcer magnitude do not appear to be functionally equivalent. Discrepant results of experiments comprised of between-condition and experimenter-controlled changes in magnitude seem to support that claim (Catania, 1963; Reed \& Wright, 1988). As posited above, the different schedules of reinforcement used to maintain behavior in each of those experiments may account for some of the discrepancies. Namely, behavior maintained under VR and VI schedules increased and decreased respectively as a function of increased numbers of reinforcers in the Reed and Wright (1988) and Reed (1991) experiments, but VI responding did not change systematically in the Catania (1963) experiment. The methods of reinforcer magnitude manipulation used in the two former experiments involved a 100-ms delay between pellet deliveries during conditions with more than one pellet delivered per IRI, which introduces an unevaluated variable. Specifically, the number of reinforcer onset periods per IRI covaried with the number of pellets earned in a condition. A delay between successive pellet deliveries necessarily meant that the magazine was operated a greater number of times per IRI during phases with more pellets than those with fewer pellets, and the conditioned reinforcers that may have existed as a byproduct of magazine operation occurred more during those phases as well. The inclusion of blackout periods between successive reinforcer deliveries is not uncommon in the reinforcer magnitude literature, nor is it exclusive to experiments manipulating the number of reinforcers. Landon et al. (2003) used blackouts between successive hopper presentations, and they also found that between-condition changes in reinforcer duration controlled response rate /choice. Their procedure, however, also involved response-correlated changes in magnitude, so it is impossible to assess the impact of the 
blackout periods and conditioned reinforcement in that experiment in the absence of responsecorrelated changes.

Researchers who have evaluated between-session and experimenter-controlled changes in reinforcer duration typically have done so with a single period of access to food per IRI (Catania, 1963) such that the number of hopper operations and conditioned reinforcers present in each IRI were held constant across conditions. The Landon et al. (2003) experiment is an exception, but in their procedure the total duration of access earned for each alternative was not constant. Thus, the relative effects of reinforcer duration and number of hopper presentations were not separated. To do so, one could design an experiment wherein one response alternative produces multiple, successive hopper presentations that, combined, produce a total of $\mathrm{X}$ s cumulative access to reinforcement, and another response alternative produces the same cumulative duration of access (X s) in a single hopper presentation. If the number of hopper presentations and the conditioned reinforcers that may be associated with them are sufficient to produce a magnitude effect, then choice responding should favor the former response alternative.

Another variable that may contribute to the functional inequivalence of reinforcer duration and numbers of reinforcers is the amount of food consumed per IRI. That is, the amount of food is directly manipulated when the number of pellets is the independent variable, but the use of common laboratory equipment makes it difficult to regulate (and to measure) the amount of food consumed when reinforcement duration is the independent variable (Epstein, 1985). Pigeon experiments in particular tend to involve manipulations of duration of access to food by controlling the amount of time that a hopper is presented per reinforcement period, and it is reasonably assumed that the amount of food consumed is a linear function of the duration of hopper presentation. That assumption, however, has been empirically demonstrated to be 
incorrect under certain conditions. That is, some feeder models, like the Lehigh Valley

Electronics feeder with a one-piece magazine, have been shown to produce linear feeding

functions as a result of hopper duration, but others, namely the older Ralph Gerbrands Companydesigned two-piece models, produce asymptotic relations between feeding and hopper duration (Epstein, 1985). Using the latter, Epstein (1985) found the amount eaten per hopper presentation increased proportionally to hopper duration up to $7 \mathrm{~s}$ of access, at which time the amount eaten reached asymptote. Seven-s access is a value that is frequently exceeded in reinforcer duration experiments (see Lendenmann et al. 1981; Neuringer, 1967 for examples), but the type of feeder used in reinforcer duration experiments is not always reported. Thus, reports of reinforcer duration experiments that did not include the type of feeder used potentially suffer from different programmed durations of access to food (the amount of time the hopper is raised, granting access to food) and obtained durations of access to food (the amount of time the animal spends eating the food in the hopper while it is raised.)

Food consumption per session has not been reported in the reinforcement magnitude literature but is likely a controlling variable in experiments that program changes in reinforcer magnitude by varying reinforcer duration. One exception is Hall and Lattal (1999), who evaluated rates of pigeon key-pecking responses when reward density was manipulated between sessions and independently of responding. Reward density was defined as "the percentage of total baseline eating duration per hopper access divided by the reinforcement schedule value" (Hall \& Lattal, 1999, p. 342), representing a multiplicative combination of reinforcement rate and duration of access to food. Reinforcer density was manipulated across phases of the experiment by changing (a) the VI value, (b) the duration of access to food, and/or (c) both. VI response rates increased as a function of reward density according to a hyperbolic function for 
three of four pigeons. Additionally, eating efficiency was evaluated by measuring the amount of food eaten in grams per hopper presentation and dividing that value by the duration of access. Eating efficiency was lower in conditions that provided longer hopper durations, and, thus, programmed reward densities were often different from obtained densities. To date, no other experimenters have provided a precise measurement of food consumption during reinforcer duration experiments. In the absence of those data, empirical investigations of behavior change as a function of food consumption are necessary to establish their role in the reinforcer duration literature.

\section{Statement of the Problem}

The conceptual foundations of behavior analysis as well as the empirical research on the parameters of reinforcement suggest that changes in reinforcer magnitude should systematically change behavior in kind (Bonem \& Crossman, 1988). That said, behavior change produced by manipulations of reinforcer magnitude varies considerably depending on a number of factors including (a) the dimension of reinforcer magnitude that is manipulated, (b) whether those manipulations occur within sessions or between conditions, and (c) whether magnitude variations are programmed so that they are correlated with or occur independently of behavior. Bonem and Crossman (1988) noted that "it is clear from the literature that these procedural variables mentioned above [magnitude contingencies or the lack thereof and changes in magnitude programmed within sessions or between conditions] may be manipulated to alter its [reinforcer magnitude's] effectiveness" (Bonem \& Crossman, 1988, p. 348). Despite that, few direct comparisons of these procedures have been conducted to evaluate their relative efficacy in generating behavior change as a function of reinforcer magnitude. Only one procedure, responsecorrelated changes in magnitude that occur within sessions, has produced reliable differential 
magnitude effects, but the mixed results reported by experimenters using the other procedures (response-correlated changes occurring between-conditions, experimenter-controlled changes occurring within-sessions, and experimenter-controlled changes occurring between-conditions) indicate that there is more to learn about the controlling variables of reinforcer magnitude under those conditions. Comparisons of these procedures and the behavior change they produce as a function of reinforcer magnitude are key to establishing a reliable data base on reinforcer magnitude and incorporating the parameter into a cohesive theory of reinforcement (Bonem \& Crossman, 1988).

Catania (1963) and Neuringer (1967) are among the few direct comparisons of these procedures. Catania (1963) used concurrent and single schedules to compare response-correlated changes in magnitude that occurred within sessions to experimenter-controlled changes in magnitude that occurred between conditions respectively. Neuringer (1967) used a concurrentchain schedule to evaluate behavior change as a function of response-correlated changes in magnitude in initial links and experimenter-controlled changes in magnitude in terminal links. No single experiment has directly compared control by reinforcer magnitude using all four of these procedures. These comparisons can be made using the elegant procedure described by Neuringer (1967) by arranging response-contingent and experimenter-controlled changes in magnitude in initial and terminal links respectively as Neuringer (1967) did, but also arranging within-sessions and between-conditions changes in magnitude as Catania (1963) did. These analyses would make up part of a systematic line of research dedicated to evaluating the efficacy of each of the above procedures.

The purpose of the current experiments was to compare the control of responding by reinforcer magnitude when reinforcer duration (Experiment 1) or temporal compositions of 
reinforcement (Experiment 2) varied (a) within sessions and correlated to responding, (b) within sessions and independently of responding, (c) between conditions and correlated to responding, and (d) between conditions and independently of responding. Direct comparisons of all four procedural variables may reveal systematicity in the behavior change or a lack thereof that occurs within and between conditions, and thereby play a key role in establishing the reliable database on reinforcer magnitude that Bonem and Crossman (1988) called for decades ago.

\section{Experiment 1}

Experiment 1 was designed to evaluate and compare directly the relative efficacy of each of the four procedures discussed above to produce control by reinforcer magnitude. Procedures were similar to Neuringer (1967), with equal reinforcer durations available for completing each chain during baseline conditions (2:2) and unequal reinforcer durations for each chain during experimental conditions (4:2 and 8:2). Food consumption (measured in grams) was recorded each session to investigate the role of eating efficiency in control by reinforcer duration.

\section{Method}

\section{Subjects}

Three adult male White Carneau pigeons were maintained at approximately 80 percent of their respective free feeding weights with food earned during experimental sessions and supplemental food provided in a vivarium post session. Each pigeon had previous experience responding on schedules of positive reinforcement, so shaping key-pecking responses was not necessary. Pigeons were housed individually in a vivarium with a 12-hour light/dark cycle and free access to water.

\section{Apparatus}


A sound-attenuating operant conditioning with a work area of $32 \mathrm{~cm}$ high, $32 \mathrm{~cm}$ long, and $38 \mathrm{~cm}$ wide was used. The chamber was illuminated at all times within a session except during reinforcement by a house light located behind a $4 \mathrm{~cm}$ by $4 \mathrm{~cm}$ aperture on the bottom right side of the response panel, $4 \mathrm{~cm}$ from the floor and $4 \mathrm{~cm}$ from the right wall. An aluminum work panel comprised one wall of the chamber, displaying two response keys. Each $2 \mathrm{~cm}$ diameter key was located $22 \mathrm{~cm}$ from the chamber floor. The response keys were located $8 \mathrm{~cm}$ from the midline of the response panel on each side. The left and right keys were transilluminated by white, green, or red lights located directly behind them. Response were be operated by a force of approximately $0.15 \mathrm{~N}$. Reinforcement was available in a Lehigh Valley Electronics food magazine located behind a $5 \mathrm{~cm}$ by $4.5 \mathrm{~cm}$ rectangular feeder aperture at the midline of the work panel, $15 \mathrm{~cm}$ from the chamber floor. It was raised into the aperture during reinforcement periods to provide access to pellets. A white light illuminated the hopper and the pellets in it during reinforcement periods. A photocell was placed on the inside of the aperture to detect hopper entry and exit by the pigeon. A fan was located behind the work panel which remained on for the duration of each session to provide white noise and mask extraneous auditory stimuli. MedPC software was operated on a computer in an adjacent room to control the experiment.

\section{Initial Training}

Each pigeon was exposed to several sessions of initial training. Initial training sessions alternated daily between (a) concurrent VI VI and (b) concurrent VR VR schedules. During VI sessions, both keys were white, and responses were reinforced according a concurrent VI 10-s VI 10-s schedule during the first session. The VI value increased by $10 \mathrm{~s}$ in each subsequent session until the terminal VI value of $30 \mathrm{~s}$ was reached and maintained. During VR sessions, the left key 
was lit red and the right key was lit green; key pecks on each were reinforced according to a concurrent VR 10 VR 10 schedule during the first session. The VR value increased by 10 in each subsequent VR session until a terminal VR value of 30 was reached and maintained. All variable schedules were constructed using the distribution described by Fleshler and Hoffman (1962). Reinforcement periods in both VI and VR training sessions were 3-s access to food pellets, with the reinforcement time cycle starting when the photocell beam in the hopper was broken (i.e. the pigeon's head enters the hopper.) Following this initial training, the experiment proper began.

\section{Procedure}

Sessions occurred five days a week at approximately the same time each day for each pigeon unless a given pigeon was under or over their target weight by more than $3 \%$ of said target. Each session began with a 180-s blackout period, during which the chamber was dark and the response keys were inoperative. Sessions ended after 60 reinforcers were delivered or after 80 min of session time elapsed (excluding reinforcement periods), whichever occurred first. Sessions consisted of 24 forced-choice trials and 36 free-choice trials for all. Each session followed the pattern of 4 forced-choice trials followed by 6 free-choice trials until the maximum number of reinforcers were earned. The purpose of this arrangement was to ensure exposure to each value of the independent variable before free-choice trials were presented and to provide multiple exposures to those contingencies throughout each session.

Forced-choice trials began with one of the two keys transilluminated by a white light. Responses to the active key were reinforced with the initiation of the terminal link according to a VI 30-s schedule. The active terminal link arranged a VR 30 schedule on the active key. When the left key was active during terminal links it was red. When the right key was active during terminal links it was green. For Pigeons 10247 and 10028, completion of the terminal link on the 
left key resulted in either 2-, 4-, or 8-s durations of access to pellets depending on the condition of the experiment, and completion of the terminal link on the right key always resulted in 2-s access to pellets. For Pigeon 20542, completion of the terminal link on the left key always resulted in 2-s access to pellets, and completion of the terminal link on the right key resulted in either 2-, 4-, or 8-s durations of access to pellets depending on the condition of the experiment. From this point on, the chain that produced 2-s duration of access throughout the experiment will be referred to as the smaller chain, and the chain that produced 2-, 4-, or 8-s duration of access depending on the condition of the experiment will be referred to as the larger chain. Table 1 shows the specific reinforcer durations available for completing each chain in each condition.

The procedure for free-choice trials is shown in Figure 1. Free-choice trials were identical to forced-choice trials except that both the left and right key were active during initial links. That is, a concurrent VI 30-s VI 30-s schedule operated across the two keys and the first response that occurred after the scheduled interval elapsed was be reinforced with the presentation of the terminal link on the selected key. Simultaneously, the other key and associated key light were deactivated for the remainder of the trial. VI schedules were independent (i.e. each schedule operated on a different interval during each trial) and non-resetting (i.e. earning a terminal link on Key A did not reset the interval timer for Key B in the next trial, but both timers were paused during terminal links). The purpose of free-choice trials was to measure preference (i.e., response allocation) between the larger and smaller chains when the reinforcer magnitude was equal during the baseline (2:2) condition and unequal during experimental (4:2 and 8:2) conditions.

Each reinforcer-cycle duration commenced only when the pigeon's head entered the food hopper, thereby breaking a photocell that initiated the reinforcement-cycle timer. The reinforcement-cycle timer only incremented whenever the photocell remained broken (i.e. the 
pigeon's head remained in the hopper) and would pause whenever the photocell was unbroken. Due to limitations of the apparatus, a limited hold of $30 \mathrm{~s}$ was placed on each reinforcement period. That is, during a given reinforcement period the pigeon was allowed up to 30 s to spend $\mathrm{X}$ $\mathrm{s}$ with their head in the hopper ( $\mathrm{X}$ being the programmed reinforcer duration, the value of which depended on the active key and the condition of the experiment). Reinforcement periods ended whenever either X s passed with the photocell broken or $30 \mathrm{~s}$ passed, whichever occurred first. This limited hold timer was implemented to prevent the hopper solenoids from burning out in the event that a pigeon would not enter the hopper, which would otherwise result in the hopper remaining raised indefinitely and, ultimately, toasting the hopper.

The conditions of Experiment 1 and reinforcer durations associated with each chained schedule appear in Table 1. The label for each condition was derived from the relative ratio of reinforcer duration available for the larger and smaller chains during each condition. That is, during the 2:2 condition, reinforcer durations were equal ( $2 \mathrm{~s}$ each) across the two keys. The 4:2 condition represents a twofold increase in the reinforcer duration on the larger chain $(4 \mathrm{~s})$ compared to the right key ( $2 \mathrm{~s})$, and so on for the remaining conditions. The experiment began and ended with the baseline, 2:2, condition for all pigeons. Pigeons 10247 and 10028 were recieved the experimental conditions in ascending order $(2: 2,4: 2,8: 2$, and 2:2) and Pigeon 20542 received them in descending order $(2: 2,8: 2,4: 2$, and 2:2). The number of sessions conducted in each condition for each pigeon is shown in Table 2. Conditions were changed after a minimum of 10 sessions and only when response rates in initial and terminal links for each key reached stability according to a statistical criterion or after 30 sessions were conducted, whichever occurred first. The 30-session limit was met only once in Experiment 1 during the initial baseline condition for Pigeon 20542. The stability criterion was based on the average 
response rates of the most recent 6 sessions and the average response rates for each block of 3 sessions therein. If the average for each block of 3 sessions did not deviate by the total 6-session average by more than $5 \%$ (upwards or downwards) then the stability criterion was met. The difference of the total 6-session average and 100 was added to the each of the averages for the 3 session blocks to account for differences in response rates that would make the statistical criterion differentially relaxed or strict depending on the unadjusted response rates. Ergo, adjusted response rate averages for each of the 3-session blocks needed to be between 95 and 105 (adjusted) responses per minute to satisfy the stability criterion.

\section{Results}

Data for free-choice trials including response rates during initial links, run rates during terminal links, and food consumed per session are show in Figure 2. Average PRP (calculated as the cumulative duration of PRP in a given link of a given chain in a session/the number of reinforcers earned on said link during said session) data for each link of the concurrent-chain schedule are shown in Figure 3. The number of terminal link entries for each chain are shown in figure 8 , and the forced-choice trail data are shown in figure 9.

\section{Response-Correlated Changes in Magnitude}

Initial-link response rates for the last 6 sessions of each condition are shown in the top row of Figure 2. Note that Pigeon 20542 received the conditions in descending order and had the larger chain on the right key, whereas the others (Pigeons 10247 and 10028) received the conditions in ascending order and had the larger chain on the left key. Pigeon 10247's initial link-response rates varied both within-sessions and between-conditions. There was a preference for the larger chain in each phase, indicating control by reinforcer magnitude as a function of 
within-sessions, response-correlated changes in magnitude. Additionally, a systematic increase in the initial-link response rates occurred between the 4:2 and 8:2 conditions on the larger chain indicating control by reinforcer magnitude as a function of between-conditions, responsecorrelated changes in magnitude. However, neither of the observed changes reversed to initial baseline levels whenever the baseline condition was reinstated. Instead, initial-link response rates on the larger chain continued to increase in the final baseline relative to previous phases. The lack of a reversal prevents a robust conclusion that the changes in Pigeon 10247's initial-link response rates were truly a function of response-correlated changes in reinforcer magnitude. No systematic change in initial-link response rates occurred for Pigeons 10028 or 20542 within sessions or between conditions as a function of response-correlated changes in magnitude. Pigeon 10028 preferred the smaller chain (as indicated by relatively high response rates on that chain relative to the larger chain) throughout the experiment. A level change (i.e. a relative change in response rates compared to those observed in the previous condition) in Pigeon 10028's initial-link response rates occurred across the 4:2 and 8:2 phases for both the left and right keys. Initial-link response rates on each chain returned to levels observed in the initial baseline and the 4:2 phase near the conclusion of final baseline phase, indicating control by between-conditions, response-correlated changes in magnitude in favor of the smaller chain. Pigeon 20542's initial-link response rates were relatively variable throughout the experiment compared to the other pigeons. The source of this variability was never identified, but it did not appear to be a function of the independent variable. That is, no systematic changes in Pigeon 20542's initial-link response rates were apparent as a function of response-correlated changes in magnitude. 
Initial-link average PRPs (hereafter referred to as initial PRP) for the last 6 sessions of each condition are shown in the top row of Figure 3. No systematic changes in initial PRP were observed for any of the Pigeons in Experiment 1. Relatively long initial PRPs occurred on the larger chain relative to the smaller chain for Pigeon 10247 during the 4:2 Condition but did not replicate during the 8:2 Condition. Similarly, relatively long initial PRPs occurred on the smaller chain relative to the larger chain for Pigeon 10028 throughout the initial baseline and experimental conditions, but those differences were not reversed during the final baseline condition. Finally, Pigeon 20542's initial PRPs were similar on the larger and smaller chains throughout experimental phases. In summary, within-sessions, response-correlated changes in reinforcer magnitude did not appear to control initial PRP in a systematic fashion. Betweenconditions, response correlated changes in magnitude did not appear to control initial PRP systematically either. Pigeon 10247's initial PRP was slightly longer on the larger chain relative to the smaller chain during experimental phases but did not reverse during the final baseline. Pigeon 10028's initial PRPs on the larger chain had a wide range of variability during the initial baseline (including values between 3 - and 23 s), making behavior change during experimental phases relatively difficult to interpret. That said, Pigeon 10028's initial PRP was similar across each of the experimental phases and remained at that level during the final baseline. Thus, between-conditions changes in Pigeon 10028's initial PRPs were not likely a function of the response-correlated changes in magnitude. Pigeon 20542's initial PRPs were similar on both the larger and smaller chains throughout the initial baseline and experimental phases of the experiment. Slight differences in initial PRP on the larger and smaller chains occurred during the final baseline phase, but those changes were not contiguous with changes in reinforcer magnitude and thus did not appear to be systematic. 


\section{Experimenter-Controlled Changes in Magnitude}

Terminal-link run rates (calculated as total responses in the terminal links divided by total time in the terminal links minus the cumulative PRP in terminal links) for the last 6 sessions of each condition are shown in the middle row of Figure 2. No systematic changes in terminal-link run rates were observed for any of the pigeons in Experiment 1. Terminal-link run rates on the larger and smaller chains were similar within each condition, indicating a lack of control by within-sessions, experimenter-controlled changes in magnitude. Additionally, no systematic increases or decreases in terminal-link run rates occurred across the experimental phases for Pigeons 10247 and 10028, indicating that between-sessions, experimenter-controlled changes in magnitude were insufficient to produce control by magnitude as well. There was a systematic increase in Pigeon 20542's terminal-link run rates on the larger chain throughout the experiment, but the trend did not reverse when baseline conditions were reinstated, and thus it may not be a function of the independent variable.

Terminal-link average PRPs (hereafter referred to as terminal PRP) for the last 6 sessions of each phase are shown in the bottom row of Figure 3. Terminal PRP was similar across the larger and smaller chains for Pigeon 10247 throughout the experimental phases, and thus were not changed as a function of within-session, experimenter-controlled changes in magnitude. Pigeons 10028 and 20542, however, had longer terminal PRPs on the larger chain relative to the those of the smaller chains throughout the experiment, which may indicate control by experimenter-controlled, within sessions changes in magnitude. Those differences, however, did not reverse during the final baseline condition and were, thus, not likely a function of withinsessions, experimenter-controlled changes in magnitude. Similarly, Pigeon 10247's terminal PRPs did not vary between conditions and thus were not changed as a function of between- 
conditions, experimenter-controlled changes in magnitude. Pigeon 10028's terminal PRP on the larger chain decreased during experimental phases relative to the initial baseline. There was a slight reversal of Pigeon 10028's terminal PRP on the larger chain, but the values observed did not return to original baseline levels nor did they exceed the range of variability observed during experimental phases; thus, those changes were not likely a function of experimenter-controlled, between conditions changes in magnitude. Pigeon 20542's terminal PRPs decreased during experiential phases relative to the initial baseline but did not reverse when the baseline conditions were reinstated. In summary, no systematic changes in terminal PRP occurred as a function of experimenter-controlled changes in magnitude, regardless of whether those changes occurred within sessions or between conditions.

\section{Programmed Reinforcer Duration and Food Consumption}

Food consumed per session is shown in grams for the last 6 sessions of each condition on the bottom row of Figure 2. Food consumed did not vary systematically across phases for Pigeons 10247 or 10028. Pigeon 20542's food consumption appeared to increase during experimental phases relative to baseline phases, but in terms of absolute value the changes were minimal. In addition to the measure of food consumption, the number of incomplete reinforcers (i.e., those that ended when the limited hold timer elapsed rather than the programmed reinforcer duration timer) was recorded for each session. As a brief reminder, if the 30-s limited-hold timer elapsed before the programmed reinforcer duration timer, then the reinforcer period would end before the pigeon could not contact the full programmed reinforcer duration. Those data are shown in Figure 4. Pigeon 10247's reinforcement periods rarely ended due to the limited hold timer regardless of the condition, indicating a relatively high level of eating efficiency compared to the other Pigeons in Experiment 1. Pigeons 10028 and 20542 contacted the limited-hold 
consequence more frequently than Pigeon 10247 in each condition, most markedly so on the larger chain. Those differences indicate a relatively low level of eating efficiency for Pigeons 10028 and 20542 during longer durations of access to reinforcement relative to Pigeon 10247. To elaborate, Pigeon 10247 regularly contacted the programmed reinforcer duration in each phase of the experiment, and the others rarely contacted the programmed reinforcer duration during experimental phases.

\section{Discussion}

\section{Response-Correlated Changes in Magnitude}

If response-correlated changes in magnitude were sufficient to produce control by reinforcer magnitude, then a preference for either the larger magnitude or smaller chain would be evident in the initial-link response rates and/or PRP during experimental phases. Although preferences for an individual chain were evident during experimental phases in two out of three pigeons (Pigeon 10247 preferred the larger chain, Pigeon 10028 preferred the smaller chain, and Pigeon 20542 was seemingly indifferent), said preferences did not reverse during the final return to baseline conditions when the chains produced equivalent durations of access to food. Pigeon 10247 's preference for the larger chain during the final baseline might be explained by behavioral history. That is, because the larger reinforcer was always available on the left key throughout the experimental phases, a stable side bias was developed over time. To reverse this side bias, and thus demonstrate experimental control, it would be necessary to conduct a greater number of sessions in the final baseline condition. To further obfuscate matters, Pigeon 10028's preference for the smaller chain would not have been predicted given the findings of similar experiments wherein response-correlated changes in magnitude reliably produced preferences for response options that were correlated to relatively large reinforcers (Buskist et al., 1988; Catania, 
1963; Gentry \& Eskew; 1984; Neuringer, 1967). Taken together with the behavior change observed with Pigeon 10247 in the current experiment, these discrepant findings call into question Bonem and Crossman's assertion that response-correlated changes in magnitude are sufficient to control choice responding in favor of larger magnitude reinforcers (1988). It is unclear if the apparent preference Pigeon 10028 showed for the smaller chain was a function of the reinforcer magnitude or some other source of bias. One possible source of Pigeon 10028's preference for the smaller chain is the location of the key on which it was scheduled.

Specifically, the smaller chain was located on the right-hand side of the operant chamber, which also happened to be the side of the box furthest from the chamber door. It has been speculated that certain subjects develop a bias against the key closest to the door because that is where they are placed into and removed from the chamber, which could be a potentially aversive event. This hypothesis could be tested experimentally using one operant chamber that opens from the side and another that opens from the top but are otherwise identical. If side bias is present or stronger in the former chamber, then the bias may be due to or enhanced by the construction of the chamber.

If within-sessions changes in magnitude were necessary to produce control by responsecorrelated changes in magnitude, then preferences would be evident in the initial-link response rates of each experimental phase, but no level change in those response rates or PRP durations would occur between the 4:2 and 8:2 conditions. Level changes in both Pigeon 10247's and Pigeon 10028's initial-link response rates occurred between the 4:2 and 8:2 Conditions. Specifically, response rates on the preferred chain increased relative to the previous phase when the reinforcer magnitude was increased on the larger chain. The level changes present in those initial-link response rates indicated that within-sessions changes in magnitude were not strictly 
necessary to produce behavior change as a function of reinforcer magnitude. Lastly, if betweenconditions changes in magnitude were sufficient to produce control by response-correlated changes in magnitude, then a level change in the initial-link response rates and/or PRP for the preferred key would occur across the 4:2 and 8:2 conditions for all three pigeons. Such level changes were present two out of three subjects; therefore between-conditions changes in magnitude were not sufficient to produce control by response-correlated changes in magnitude in isolation.

\section{Experimenter-Controlled Changes in Magnitude}

If experimenter-controlled changes in magnitude were sufficient to control terminal-link responding as a function of reinforcer magnitude, then terminal-link run rates and/or PRP would differ systematically on the larger and smaller chains in each experimental phase. If withinsession changes in magnitude were necessary to produce control by experimenter-controlled changes in magnitude, then terminal-link run rates and/or PRP would be relatively high on one chain compared to the other in a given experimental phase, but no level change in those run rates or PRP would occur across the 4:2 and 8:2 conditions. Alternatively, if between-conditions changes in magnitude were sufficient to produce control by experimenter-controlled changes in magnitude, then a level change in the terminal-link run rates and/or PRP on the larger chain would occur across the 4:2 and 8:2 conditions. No systematic differences in terminal-link run rates were present between the larger and smaller chains for Pigeons 10247 or 10028 . Pigeon 20542's terminal-link run rates were slightly higher on the larger chain relative to the smaller chain during experimental conditions but said difference did not change between conditions nor did it reverse to original baseline levels during the final baseline. Experimenter-controlled changes in reinforcer magnitude were insufficient to control terminal-link responding regardless 
of whether they occurred within sessions or between conditions. These findings are consistent with much of the literature discussed in the introduction. That is, few experimenters have reported systematic changes in behavior as a function of magnitude whenever magnitude varied without correlation to the behavior producing the reinforcer (Bonem \& Crossman, 1988). The majority of those that have were different from the current experiment in terms of the dimension of magnitude manipulated. Specifically, Reed (1991) and Reed and Wright (1988) manipulated the number of reinforcers per IRI rather than the duration of access to reinforcers. With those experiments in mind, the current findings provide additional evidence that the different dimensions of reinforcer magnitude may not be functionally equivalent.

\section{Food Consumption and Incomplete Reinforcers}

The behavior change in Pigeon 10247's initial-link response rates (preference for the larger chain) is consistent with the literature on response-correlated, within-sessions changes in magnitude (Buskist et al., 1988; Catania, 1963; Gentry \& Eskew; 1984; Neuringer, 1967). Pigeon 10028's preference for the smaller chain and Pigeon 20542's apparent lack of general behavior change as a function of magnitude may be accounted for, to some undetermined degree, by the time the animal spent contacting the reinforcer each trial. That is, although systematic behavior change as a function of response-correlated changes in magnitude did not appear to be functionally related to the amount of food consumed per session, there was a functional relation between preference for the larger chain and the number of incomplete reinforcers per session. No systematic behavior change was observed for those pigeons that regularly missed the window for

food consumption during experimental phases (Pigeons 10028 and 20542), but behavior change was observed both within sessions and between conditions for the pigeon that regularly contacted the programmed reinforcer duration (Pigeon 10247). That said, Pigeon 10247's 
behavior change did not reverse during the final 2:2 Condition, and thus robust experimental control was not demonstrated in that case. However, it is likely that the behavior did not reverse due to a lasting side bias that, itself, was functionally related to increases in reinforcer magnitude. Despite those limitations, measuring the number of reinforcers that ended due to the limited hold timer per session helped to illuminate possible systematicity in the present findings. Future experimenters would be wise to include such measurements, particularly those that can describe the consummatory chain such as duration of time spent contacting the reinforcer per trial and food consumed per session. Using those values, one can generate a quotient to more accurately describe eating efficiency; doing so may be useful in accounting for some of the discrepant findings that are so common to the reinforcer magnitude literature.

\section{Experiment 2}

As discussed in the introduction, reinforcement duration has been manipulated in two ways in the study of reinforcer magnitude: by presenting reinforcers of different total duration at different times (e.g., Catania, 1963) or by presenting sequentially different numbers of hopper presentations of the same duration (e.g., Landon et al., 2003). These different methods of manipulation are not likely to have identical functions, and there is some evidence that different temporal arrangements of sequential hopper presentations produced differential control of behavior. Namely, Shull et al. (1981) evaluated behavior maintained by a concurrent schedule with a changeover-key procedure wherein a green key light alternative arranged a single reinforcer for key pecking according to a VI 2-min schedule and a red key light alternative arranged multiple hopper presentations according to an FI 30-s schedule. Both red and green components lasted for $240 \mathrm{~s}$ when selected. During the red alternative, the first reinforcer was scheduled after $30 \mathrm{~s}$ (the FI $30 \mathrm{~s}$ ), followed by, in different conditions, two or three more 
successive, response-dependent reinforcers in different temporal relations to the first. During conditions wherein the number of reinforcers, and hence the magnitude, were held constant, the number of switches to (preference for) the red key varied with the temporal placement of those reinforcers. Specifically, the rate of switching to red increased when the reinforcer deliveries were relatively proximal in time compared to whenever they were delayed relative to one another. This finding suggests that, with total reinforcer duration and number of hopper presentations held constant, unequal temporal distributions of reinforcers can differentially control behavior. These three experiments (i.e., Catania, 1963; Landon et al., 2003; Shull et al., 1981) taken together raise the question of whether and how different distributions of the sametotal duration reinforcer might affect choice, response rates, and PRP durations. Additionally, like manipulations of the number of reinforcers (Reed, 1991; Reed \& Wright, 1988), manipulations of sequential hopper presentations necessarily involve presenting greater numbers of putative conditioned reinforcers per IRI when the number of hopper presentations is increased. The relative effects of conditioned reinforcers have not been isolated from the effects of different reinforcer durations during those experiments (Landon et al., 2003). Thus, the second experiment was an evaluation of behavior change as a function of the number of successive hopper presentations per IRI when total reinforcement duration per IRI was held constant.

\section{Method}

\section{Subjects and Apparatus}

The 3 pigeons used in Experiment 2 were similar in housing, age, sex, experience, and diet to those described in Experiment 1. The apparatus was also the same as in the one described in Experiment 1. 


\section{Procedure}

The procedure was identical to that described in Experiment 1, with the following exceptions. For Pigeons 11863, and 13715 sessions ended after 60 reinforcers were delivered or after 80 min of session time elapsed (excluding reinforcement periods), whichever occurred first. Pigeon 9553's sessions ended after 50 reinforcers were delivered or after $80 \mathrm{~min}$ of session time elapsed, whichever occurred first. This change for Pigeon 9553 was predicated on regular fluctuations of the animal's weight, which frequently exceeded the target weight when 60 reinforcers were allowed. Pigeon 9553's weight became relatively stable once the number of reinforcers per session was reduced to 50. Sessions consisted of 24 forced-choice trials and 36 free-choice trials for all pigeons except for Pigeon 9553. Pigeon 9553's sessions consisted of 20 forced-choice trials and 30 free-choice trials as a byproduct of the change in overall reinforcers per session described above.

The total programmed reinforcer duration available for completing each chain was equal to $8 \mathrm{~s}$ per IRI in each condition, but the number and duration of sequential hopper presentations per IRI varied on the segmented chain (described below) between conditions. The procedure for free-choice trials in Experiment 2 is shown in Figure 5. A single 8-s hopper presentation occurred when the nonsegmented chain was completed throughout each condition, and either one 8-, two 4-, or four 2-s hopper presentations occurred when the segmented chain was completed, depending on the condition. The number and duration of hopper presentations for completing the segmented chain are shown in Table 3. The label for each condition was derived from the relative ratio of hopper presentations per IRI for completing the segmented and nonsegmented chains in each condition. Each successive hopper presentation was separated by a .5-s blackout, which necessarily extends the total duration of the reinforcement period relative to the 
nonsegmented chain. To control for this, a blackout period followed reinforcement periods on the nonsegmented chain that was equal in duration to the total blackout duration per IRI for the segmented chain in each condition. Conditions were changed according to the same stability criterion described for Experiment 1 and occurred in the same orders described in Experiment 1 as well. The 30-session limit was met only once in Experiment 2 during the initial baseline phase for Pigeon 9553. Two pigeons (11863 and 13715) received conditions in ascending order (1:1, $2: 1,4: 1$, and $1: 1)$ and the remaining pigeon (9553) received them in descending order $(1: 1,4: 1$, 2:1, and 1:1). The number of sessions conducted in each phase for each Pigeon is shown in Table 4.

Dependent measures were the same as in Experiment 1 with one exception. That is, the counter for incomplete reinforcers (i.e. those that ended due to the limited hold timer rather than the programmed reinforcer duration timer) was not included. As noted in the Experiment 1 method and discussion, that measure was included to further investigate a curious finding with Pigeon 10028 and thus was not included at the beginning of either experiment. At that point in time, Experiment 2 was nearly complete. Including a new measure at that time would not have been fruitful because there would not have been enough data to make meaningful comparisons.

\section{Results}

The findings of Experiment 2 are shown in Figures 6, 7, 8, and 10 and are discussed below in terms of the procedures used to manipulate magnitude.

\section{Response-Correlated Changes in the Temporal Distribution of Reinforcement}

Initial-link response rates for the last 6 sessions of each condition are shown in the top row of Figure 6. Note that Pigeon 9553 received the conditions in descending order and had the 
segmented chain on the right key, whereas the others (Pigeons 11863 and 13715) received the conditions in ascending order and had the segmented chain on the left key. There was a systematic change in initial-link response rates for all three pigeons both within sessions and between conditions as a function of response-correlated changes in the temporal distribution of reinforcement. That is, each pigeon preferred the segmented chain (as indicated by relatively high response rates on that key compared to the nonsegmented key) during experimental phases (i.e. 2:1 and 4:1) indicating control of response rates in initial links by response-correlated changes in the temporal distribution of reinforcement that occurred within sessions. Additionally, level changes in the initial-link response rates on the segmented chain occurred across experimental phases for all three pigeons indicating control by response-correlated, betweenconditions changes in magnitude. However, initial-link response rates did not fully reverse to the original baseline levels when baseline conditions were reinstated. Despite that, systematic decreases in initial-link response rates occurred between the 4:1 and 1:1 conditions for those pigeons, leading to the conclusion that response-correlated, between-conditions changes in the temporal distribution of reinforcement were sufficient to produce control by reinforcer magnitude both within sessions and between conditions.

Initial PRP is shown on Figure 7. Initial PRPs on the segmented chain were shorter than those of the nonsegmented chain for all three pigeons, which was consistent with the preferences evident in the initial-link response rate data for each pigeon and may indicate control of initial PRP by response-correlated, within-sessions changes in the temporal distribution of reinforcement. There were no level changes in initial PRP on the segmented chain for any of the pigeons, indicating a lack of control by between-conditions, response-correlated changes in the temporal distribution of reinforcement or perhaps a floor effect (the initial PRP durations on 
segmented chains were regularly $3 \mathrm{~s}$ or less for all three pigeons.) Initial PRP varied on the nonsegmented chain between conditions for Pigeons 11863 and 13715 . These changes are likely a byproduct of shifting preferences during the experimental phases towards the segmented chain, perhaps indicating behavioral contrast, and thus may be functionally related to the independent variable as well. Initial PRPs were similar throughout the experiment for Pigeon 9553 and did not appear to change as a function of the temporal distribution of reinforcement.

\section{Experimenter-Controlled Changes in the Temporal Distribution of Reinforcement}

Terminal-link run rates for the last 6 sessions of each condition are shown in the middle row of Figure 6. No systematic changes in terminal-link run rates were observed for any of the pigeons in Experiment 2. Segmented- and nonsegmented-chain run rates were similar within each condition for Pigeons 13715 and 9553, indicating that within-sessions, experimentercontrolled changes in the temporal distribution of reinforcement were not sufficient to produce control by reinforcer magnitude. Pigeon 11863's terminal-link run rates were similar on each chain during the initial baseline phase and the $2: 1$ Condition but differed during the $4: 1$

Condition. That is, the response rates were relatively high on the segmented chain compared to the nonsegmented chain, but this level change did not reverse when baseline conditions were reinstated during the final 1:1 Condition. The lack of a reversal in the final phase indicated that the differences in segmented and nonsegmented chain run rates for Pigeon 11863 were not likely a function of changes in the temporal distribution of reinforcement. Additionally, no systematic increases or decreases in terminal-link run rates were observed across the experimental phases for Pigeons 11863 or 9553, indicating that between-sessions, experimenter-controlled changes in the temporal distribution of reinforcement were insufficient to control of terminal-link run rates once again. There was a decreasing trend in terminal-link run rates for Pigeon 13715 throughout 
the experiment, but it did not reverse when baseline conditions were reinstated and thus was not likely a function of the independent variable.

Terminal PRPs are shown in Figure 7. Terminal PRPs did not change systematically during experimental phases relative to baseline phases for any of the three pigeons throughout the experiment, indicating that no systematic changes occurred as a function of experimentercontrolled, between conditions changes in the temporal distribution of reinforcement. Pigeon 11863's terminal PRPs on the nonsegmented chain increased during 4:1 phase relative to previous conditions and continued to increase in the subsequent 1:1 phase but decreased back to baseline levels by the end of the experiment. It is unclear what led to this spike in terminal PRP for Pigeon 11863, but, because it was not contiguous with a change in the temporal distribution of reinforcement on that chain, it did not appear to be a function of the independent variable. Terminal PRP on the nonsegmented chain remained at baseline levels throughout the experiment for Pigeons 13715 and 9553, indicating no systematic change as a function of experimentercontrolled changes in the temporal distribution of reinforcement. In summary, terminal PRP did not vary as a function of experimenter-controlled changes in the temporal distribution of reinforcement, regardless of whether said changes occurred within sessions or between conditions.

\section{Food Consumption}

Food consumed per session is shown in grams for the last 6 sessions of each condition on the bottom row of Figure 6. Food consumed was greater in the experimental conditions (2:1 and $4: 1)$ relative to the baseline conditions (1:1) for all three pigeons and the difference was greatest during the 4:1 condition for all three pigeons. The observed increase in food consumed per session that occurred when the reinforcer was segmented reversed to baseline levels when during 
the final 1:1 condition for Pigeons 11863 and 13715, indicating that those changes were a function of the independent variable. Pigeon 9553's food consumption during the final baseline remained at the level observed during experimental phases, but a decreasing trend occurred in those data throughout the phase and was approaching original baseline levels at the conclusion of the experiment. The number of incomplete reinforcers (i.e., those that ended when the limited hold timer elapsed rather than the programmed reinforcer duration timer) was not recorded during Experiment 2, so those data are not available for discussion here as they were for Experiment 1.

\section{Discussion}

Similar to the report of Shull et al. (1981), Experiment 2 compared reinforcer access periods that were segmented into bins with those presented in a single, continuous access period per IRI. When compared, the two methods of manipulating reinforcer duration appear to be functionally inequivalent. Shull et al. (1981) found that the preference for concurrently available operants with equivalent durations of reinforcement was controlled by the temporal proximity of hopper presentations associated with each option. That is, the operant that produced multiple hopper presentations in close temporal proximity was preferred over the operant that produced multiple hopper presentations with relatively long delays between them even though the overall duration of access to reinforcement per trial was identical on each operant. Similarly, the pigeons in Experiment 2 of the current report preferred the segmented chain to the nonsegmented chain despite the overall reinforcer duration per IRI of each chain remaining constant throughout the experiment. Because the overall duration of reinforcement was constant in Experiment 2, the findings cannot be compared directly to the literature on reinforcer magnitude wherein the duration varied. That said, the food consumption data in Figure 6 indicated that changes in food 
consumption were functionally related to increases in the number of segments in the reinforcer access period per IRI. It can be argued that, if reinforcer magnitude is quantified by the number of pellets delivered per IRI or the duration of access to reinforcers per IRI, then it follows that the amount of food consumed per session is relevant measure of reinforcer magnitude as well. Conceptualizing reinforcer magnitude in this fashion allows comparisons between Experiment 2 and the previous literature, and the comparisons made below are predicated on that assumption.

\section{Response Correlated Changes in Temporal Distribution of Reinforcement}

If response-correlated changes in the temporal distribution of reinforcement were sufficient to produce control by reinforcer magnitude, then a preference for either the segmented or nonsegmented chain would be evident in the initial-link response rates or PRP durations during experimental phases. If within-sessions changes in the temporal distribution of reinforcement were necessary to produce control by response-correlated changes in magnitude, then preferences would be evident in the initial-link response rates of each experimental phase, but no level change in those response rates or PRP durations would occur between the 2:1 and 4:1 conditions. Alternatively, if between-conditions changes in the temporal distribution of reinforcement were sufficient to produce control by response-correlated changes in magnitude, then a level change in the initial-link response rates and/or PRP durations for the preferred key would occur across the 2:1 and 4:1 conditions. All three pigeons preferred the segmented chain as evidenced by the relatively high initial-link response rates on that key compared to the other response option, indicating that response-correlated changes in the temporal distribution of reinforcement were sufficient to produce control by reinforcer magnitude. Systematic decreases in the initial-link response rates on the segmented key occurred during the final baseline conditions relative to previous experimental phases for all three pigeons as well, but those level 
changes did not reach original baseline levels which may indicate residual side bias.

Additionally, increases in the initial-link response rates occurred for all three pigeons in proportion to the number of segments per IRI in a given phase. That is, response rates were relatively high on the segmented chain during 4:1 conditions when compared to those response rates during 2:1 conditions for all three pigeons. Therefore, both between-conditions changes and within-sessions changes in the temporal distribution of reinforcement were sufficient to produce control by magnitude, but neither were strictly necessary.

These findings are consistent with those of previous research on response-correlated changes in magnitude. Similar to Catania (1963) and Neuringer (1967), within-sessions, response-correlated changes in magnitude produced reliable and reversible preferences for the larger chain. Bonem and Crossman (1988) argued that response-correlated changes in magnitude produce reliable changes in behavior when manipulated within sessions but are relatively less reliable when manipulated between conditions. As was noted, Landon (2003) is one of few experimental reports that found systematic behavior change as a function of response-correlated, between-condition changes in magnitude. Their procedure involved segmented reinforcer access periods as well, which, as posited before, may function to increase eating efficiency. Taken together with the findings of Experiment 2 and in contrast to those of Experiment 1, it seems that eating efficiency may mediate behavior change as a function of between-conditions, responsecorrelated changes in magnitude. When eating efficiency is relatively high, response-correlated changes in magnitude appear to be more reliable regardless of whether they occur within sessions or between conditions.

\section{Experimenter-Controlled Changes in Temporal Distribution of Reinforcement}


If experimenter-controlled changes in the temporal distribution of reinforcement produced control by reinforcer magnitude, then terminal-link run rates and/or PRP durations would differ systematically on the segmented and nonsegmented chains in each experimental phase. If within-session changes in the temporal distribution of reinforcement were necessary to produce control by experimenter-controlled changes in magnitude, then terminal-link run rates and/or PRP durations would be relatively high on one chain compared to the other in a given experimental phase, but no level change in those rates or durations would occur across the $2: 1$ and 4:1 conditions. Alternatively, if between-conditions changes in the temporal distribution of reinforcement were sufficient to produce control by experimenter-controlled changes in magnitude, then a level change in the terminal-link run rates and/or PRP durations on the larger chain would occur across the 2:1 and 4:1 conditions. No systematic differences in terminal-link run rates occurred throughout the experiment for any of the pigeons. Experimenter-controlled changes in the temporal distribution of reinforcement were insufficient to produce control by reinforcer magnitude regardless of whether those changes occurred within sessions or between conditions. These findings are consistent with those of Experiment 1 and with the previous literature on experimenter-controlled changes in magnitude using duration of access to reinforcers as the independent variable (Catania, 1963; Neuringer, 1967) but inconsistent with those that manipulated number of reinforcers per IRI instead (Reed, 1991; Reed \& Wright, 1988). Based on the earlier conclusions of Bonem and Crossman (1988), the aforementioned results reported by Reed (1991) and Reed and Wright (1988) are rare exceptions to what appears to be a consistent finding in the reinforcer magnitude literature. That is, experimenter-controlled changes in reinforcer duration that occur regardless of the behavior which produces said reinforcers are generally insufficient to produce systematic behavior change. 


\section{General Discussion}

The findings of Experiments 1 and 2, at a glance, appear to be in stark contrast to one another. Little to no systematic behavior change occurred in Experiment 1 as a function of increasing reinforcer access duration, but segmented periods of access to reinforcement in Experiment 2 produced preferences for the segmented reinforcer chain for all three pigeons despite holding the overall reinforcer access duration constant. Upon further examination of those data, systematicity was revealed in the measurement of food consumption and incomplete reinforcers. That is, the pigeons that regularly contacted the programmed reinforcer duration in Experiment 1 also tended to prefer the larger chain (Pigeon 10247), but those that regularly experienced the limited hold on reinforcer delivery, and thus did not contact the programmed reinforcer duration, did not prefer the larger chain (Pigeons 10028 and 20542). What seems to distinguish the subjects and their respective behavior changes is a difference in eating efficiency. In this case, the term eating efficiency is used imprecisely to refer to a subject's overall food consumed per session in relation to the reinforcer access duration and the number of incomplete reinforcers of said session. For example, Pigeon 10247 consumed more food per session (an approximate range of 10-15g per session) and had fewer incomplete reinforcers per session than the other two Pigeons (with approximate ranges of 5-7g and 6-9g per session for Pigeons 10028 and 20542 respectively) in each phase despite having equal durations of access. Pigeon 10247 thus had a higher eating efficiency relative to the others. Likewise, food consumption of all three pigeons in Experiment 2 increased as a function of increasing the number segments in the reinforcer access period, despite the overall reinforcer duration remining the same. Thus, increases in consumption given the same period of access indicated increases in eating efficiency as well. Hall and Lattal (1999) found that eating efficiency decreased in inverse proportion to the 
overall duration of access, so perhaps segmenting the reinforcer in Experiment 2 into shorter bins prevented decreases in eating efficiency. It also is possible that increased frequencies of conditioned reinforcement associated with operating the hopper multiple times in quick succession could account for some of the changes in consummatory behavior observed in Experiment 2. Though it is unclear which variables control eating efficiency, consummatory behavior is clearly an operant and it can be controlled. Further experimentation is invited to describe the functional relations between consummatory behavior, reinforcer duration, and conditioned reinforcement.

Where previous experimenters have reported linear relations between duration of access to food and amount of food consumed (Epstein, 1985), the findings of the Experiment 1 suggest a more nuanced relation, one that varies between subjects according to variables not yet identified. In further contrast to Epstein (1985), Experiment 2 showed that consumption varies as a function of the temporal distribution of reinforcement, but not in linear relation to the overall duration of access to reinforcement per IRI. Excluding Hall and Lattal (1999), few have reported measurements of food consumption in the reinforcer magnitude literature. In the absence of replications for neither the current experiments nor Epstein (1985) it remains unclear how food consumption varies as a function of duration of access to food. Additional research is necessary to describe the consummatory chain and the variables that control it. Until a sound body of literature on reinforcer consumption is available for reference, researchers cannot design experiments to investigate reinforcer magnitude effects from an informed perspective. To remedy this, future experimenters investigating reinforcer duration would be wise to include measures of food consumption and time spent contacting the reinforcer per access period. Those measurements can be used to create an eating efficiency quotient for each session. Additionally, 
a line of descriptive research dedicated to measuring food consumption given different durations of access to reinforcement and evaluating the relations between duration of access and eating efficiency would likely be fruitful in the analysis of behavior change as a function of reinforcer magnitude.

\section{Postreinforcement Pausing}

It is noteworthy that no systematic changes in pausing behavior occurred as a function of reinforcer magnitude in either of the current experiments. Previous experimenters have found that PRP varied as a function of experimenter-controlled changes in reinforcer magnitude when magnitude alternated between components of multiple schedules (Blakely \& Schlinger, 1988; Perone \& Courtney, 1992) and when alternated between conditions of an experiment (Reed \& Wright, 1988). Terminal-link behavior did not affect reinforcer duration in the current experiments, so one might predict that PRP would differ systematically from the larger chain to the smaller chain during terminal links. However, magnitude values in the current experiments did not strictly alternate as they might in a multiple schedule, but rather were determined by initial-link responding. The lack of strict alternation prevented regular rich-to-lean transitions that were characteristic of the experiments wherein magnitude varied between components of multiple schedules (Blakely \& Schlinger, 1988; Perone \& Courtney, 1992). Rich-to-lean transitions such as these control PRP as a function of magnitude, but no such transitions were present during the current experiments, outside of the forced-choice trials. Thus, it may have been possible to produce control of PRP in the current experiments if follow-up conditions were conducted wherein the response-correlated changes in magnitude were removed and instead magnitude values alternated each trial or after some duration of time. Unlike those experiments, Reed and Wright (1988) found systematic differences in PRP as a function of between- 
conditions changes in magnitude, and without frequent rich-to-lean transitions. They, however, manipulated the number of reinforcers delivered per IRI instead of the reinforcer duration, and thus it is possible that the functional inequivalence of those dimensions is accountable for the lack of control over PRP found in the present experiments.

\section{Limitations}

As posited above, the utility of the present findings would be enhanced by more exhaustive and precise measurement of the consummatory chain. The measurement of incomplete reinforcers was included during the first experimental phase of Experiment 1 for Pigeons 10247 and 10028, instead of from the beginning of the Experiment as it was for Pigeon 20542. The inclusion of this measure was a search for the source of preference for the smaller chain present in Pigeon 10028's initial-link response rates during the 4:1 Condition. Pigeon 20542 had not yet achieved stability in the initial baseline at that time, so it was possible to include the measure before any phase changes were made. The reactive inclusion of the measure limited the number of available comparisons between experimental phases and baseline conditions for Pigeons 10247 and 10028. Number of incomplete reinforcers per session was also somewhat insufficient as a measurement. That is, the count of incomplete reinforcers shed light on eating efficiency in terms of opportunities missed but did not allow for a precise comparison of programmed duration of reinforcement with obtained duration of reinforcement. Further, the counter was not included in Experiment 2. Those data may have been useful in identifying systematicity amongst the findings, but their absence prevents valuable speculation. It is recommended that any experimenters who may replicate or extend this line of research include not only a measurement of food consumption but also a measurement of time spent in the hopper per reinforcer access period for those reasons. 


\section{Summary and Conclusions}

Bonem and Crossman (1988) called for researchers to identify conditions necessary and/or sufficient to observe behavior change given a change in reinforcer magnitude. Thus, the purpose of the present experiments was to heed this call and evaluate behavior change as a function of changing magnitude using four different procedures common within the reinforcer magnitude literature. Those procedures were response-correlated, experimenter controlled, within-sessions, and between-conditions changes in magnitude. The findings of both experiments are summarized as follows. Response-correlated changes in reinforcer magnitude controlled response rates both within sessions and between conditions, but the observed effect appeared to be mediated by eating efficiency in Experiment 1. Experimenter-controlled changes in magnitude were insufficient to produce systematic changes in neither run rate nor PRP regardless of whether the changes occurred within sessions or between conditions, and regardless of eating efficiency.

It comes as no surprise that reinforcers must be consumed in order to function as such, but the dimensions of behavior that constitute the consummatory chain vary between subjects. If these variations in consummatory behavior indeed mediate reinforcer magnitude effects, then they may well be responsible for the many inconsistencies present in the findings available in the literature. To identify systematicity in the reinforcer magnitude literature and thus create a coherent body of literature on the topic, researchers must measure the consummatory chain in detail when conducing future research. Replications of previous experiments that found systematic findings would be a fruitful addition to the literature, as would a series of descriptive research studies dedicated to evaluating the consummatory chain given different durations of access to reinforcement. It also is likely that the frequency of conditioned reinforcers associated 
with the operation of hoppers/magazines in reinforcer magnitude experiments may contribute to differences in consummatory behavior, as evidenced in those food consumption data of Experiment 2. Given descriptive literature on eating efficiency in the presence of different duration of access to food, one could make comparisons between values produced by a single period of access per IRI and those produced by multiple (i.e. segmented) periods of access per IRI. Such comparisons may reveal differences that predicate further research on the role of conditioned reinforcement in reinforcer magnitude effects. 


\section{References}

Baron, A., Mikorski, J., Schlund, M. (1992). Reinforcement magnitude and pausing on progressive-ratio schedules. Journal of the Experimental Analysis of Behavior, 58(2), $377-388$.

Blakely, E., \& Schlinger, H. (1988). Determinants of pausing under variable-ratio schedules: Reinforcer magnitude, ratio size, and schedule configuration. Journal of the Experimental Analysis of Behavior, 50(1), 65-73.

Bonem, M., \& Crossman, E. K. (1988). Elucidating the effects of reinforcement magnitude. Psychological Bulletin, 104(3), 348-362.

Buskist, W., Oliviera-Castro, J., \& Bennett R. (1988). Some effects of response-correlated increases in reinforcer magnitude on human behavior. Journal of the Experimental Analysis of Behavior, 49(1), 87-94.

Catania, C. A. (1963). Concurrent performances: A baseline for the study of reinforcement magnitude. Journal of the Experimental Analysis of Behavior, 6(2), 299-300.

Da Silva, S., \& Lattal, K. A. (2010). Why pigeons say what they do: Reinforcer magnitude and response requirement effects on say responding in say-do correspondence. Journal of the Experimental Analysis of Behavior, 93(3), 359-413.

Davison, M., \& Baum, W. M. (2003). Every reinforcer counts: Reinforcer magnitude and local preference. Journal of the Experimental Analysis of Behavior, 80(1), 95-129. 
Dougherty, D. M., \& Cherek, D. R. (1994): Effects of social context, reinforcer probability, and reinforcer magnitude on humans' choices to compete or not to compete. Journal of the Experimental Analysis of Behavior, 62(1), 133-148.

Doughty, A. H., \& Richards, J. B. (2002). Effects of reinforcer magnitude on responding under differential-reinforcement-of-low-rate schedules of rats and pigeons. Journal of the Experimental Analysis of Behavior, 78(1), 17-30.

Ettinger, R. H., McSweeney, F. K., \& Norman, W. D. (1981). Contrast and undermatching as a function of reinforcer duration and quality during multiple schedules. Journal of the Experimental Analysis of Behavior, 35(3), 271-282.

Epstein, R. (1985). Amount consumed varies as a function of feeder design. Journal of the Experimental Analysis of Behavior, 44(1), 121-125.

Fantino, E., \& Davison, M. (1983). Choice: Some quantitative relations. Journal of the Experimental Analysis of Behavior, 40(1), 1-13.

Gentry, G. D., \& Eskew, Jr., R. T. (1984). Graded differential reinforcement: Responsedependent reinforcer amount. Journal of the Experimental Analysis of Behavior, 41(1), $27-34$.

Gleeson, S., \& Lattal, K. A. (1987). Response-reinforcer relations and the maintenance of behavior. Journal of the Experimental Analysis of Behavior, 48(3), 383-393.

Hall G. A., \& Lattal, K. A. (1999). Reward density and variable-interval schedule performance in an open economy. Journal of the Experimental Analysis of Behavior, 72, 341-354. 
Herrnstein, R. J. (1970). On the law of effect. Journal of the Experimental Analysis of Behavior, 13(3), 243-266.

Keesey, R. E., \& Kling, J. W. (1961). Amount of reinforcement and free-operant responding. Journal of the Experimental Analysis of Behavior, 4, 125-132.

King, G. R., \& Logue, A. W. (1990). Humans' sensitivity to variation in reinforcer amount: Effects of the method of reinforcer delivery. Journal of the Experimental Analysis of Behavior, 53(1), 33-45.

Landon, J., Davison, M., \& Elliffe, D. (2003). Concurrent schedules: Reinforcer magnitude effects. Journal of the Experimental Analysis of Behavior, 79(3), 351-365.

Lendenmann, K. W., Myers, D. L., \& Fantino, E. (1982). Effects of reinforcer duration on responding in two-link chained interval schedules. Journal of the Experimental Analysis of Behavior, 37(2), 217-222.

McSweeney, F. K., Weatherly, J. N., \& Swindell, S. (1995). Within-session response rates when reinforcement rate is changed within each session. Journal of the Experimental Analysis of Behavior, 64(2), 237-246.

Neuringer, A. J. (1967). Effects of reinforcement magnitude on choice and rate of responding. Journal of the Experimental Analysis of Behavior, 10(5), 417-424.

Perone, M., \& Courtney, K. (1992). Fixed-ratio pausing: Joint effects of past reinforcer magnitude and stimuli correlated with upcoming magnitude. Journal of the Experimental Analysis of Behavior, 57(1), 33-46. 
Reed, P. (1991). Multiple determinants of the effects of reinforcement magnitude on free-operant response rates. Journal of the Experimental Analysis of Behavior, 55(1), 109-123.

Reed, P., \& Wright, J. E. (1988). Effects of Magnitude of Food Reinforcement on Free-Operant Response Rates. Journal of the Experimental Analysis of Behavior, 49(1), 75-85.

Schmitt, D. R. (1974). Effects of reinforcement rate and reinforcer magnitude on choice behavior of humans. Journal of the Experimental Analysis of Behavior, 21(3), 409-419.

Schwartz, B. (1969). Effects of reinforcement magnitude on pigeons' preference for different fixed-ratio schedules of reinforcement. Journal of the Experimental Analysis of Behavior, $12(2), 253-259$.

Shull, R. L., Spear D. J., \& Bryson A. E. (1981). Delay or rate of food delivery as a determiner of response rate. Journal of the Experimental Analysis of Behavior, 35(2), 129-143.

Sizemore, O. J., \& Lattal, K. A. (1978). Unsignalled delay of reinforcement in variable-interval schedules. Journal of the Experimental Analysis of Behavior, 30(2), 169-175.

Stubbs, D. A., \& Pliskoff, S. S. (1969). Concurrent responding with fixed relative rate of reinforcement. Journal of the Experimental Analysis of Behavior, 12(6), 887-895.

Williams, D. C., Saunders, K. J., \& Perone, M. (2011). Extended pausing by humans on multiple fixed-ratio schedules with varied reinforcer magnitude and response requirements. Journal of the Experimental Analysis of Behavior, 95(2), 203-220. 


\begin{tabular}{c|ccc} 
Condition & $\begin{array}{c}\text { Number of Hopper } \\
\text { Presentations Per Trial }\end{array}$ & $\begin{array}{c}\text { Reinforcer Duration for } \\
\text { the Larger Chain }\end{array}$ & $\begin{array}{c}\text { Reinforcer Duration for } \\
\text { the Smaller Chain }\end{array}$ \\
\hline \hline $2: 2$ & 1 & $2 \mathrm{~s}$ & $2 \mathrm{~s}$ \\
$4: 2$ & 1 & $4 \mathrm{~s}$ & $2 \mathrm{~s}$ \\
$8: 2$ & 1 & $8 \mathrm{~s}$ & $2 \mathrm{~s}$
\end{tabular}

Table 1. The number and duration of hopper presentations for completing each chain in each condition of Experiment 1.

\begin{tabular}{c|rrr}
\multirow{2}{*}{ Condition } & \multicolumn{3}{|c}{ Number of Sessions } \\
\cline { 2 - 3 } & 10247 & 10028 & 20542 \\
\hline \hline $2: 2$ & 19 & 18 & $30^{*}$ \\
$2: 4$ & 13 & 19 & 11 \\
$2: 8$ & 12 & 17 & 15 \\
$2: 2$ & 15 & 12 & 12
\end{tabular}

Table 2. The number of sessions conducted in each condition for each pigeon in Experiment 1.

Stability criteria were met in each condition by each pigeon excluding those indicated with *.

\begin{tabular}{c|ccc} 
Condition & $\begin{array}{c}\text { Number of Hopper } \\
\text { Presentations IRI }\end{array}$ & $\begin{array}{c}\text { Duration of Access Per } \\
\text { Hopper Presentation }\end{array}$ & $\begin{array}{c}\text { Total Duration of } \\
\text { Access Per IRI }\end{array}$ \\
\hline \hline $1: 1$ & 1 & $8 \mathrm{~s}$ & $8 \mathrm{~s}$ \\
$2: 1$ & 2 & $4 \mathrm{~s}$ & $8 \mathrm{~s}$ \\
$4: 1$ & 4 & $2 \mathrm{~s}$ & $8 \mathrm{~s}$
\end{tabular}

Table 3. The number and duration of hopper presentations for completing the segmented chain in each phase of Experiment 2. 


\begin{tabular}{c|rrr}
\multirow{2}{*}{ Condition } & \multicolumn{3}{|c}{ Number of Sessions } \\
\cline { 2 - 4 } & 11863 & 13715 & 9553 \\
\hline \hline $1: 1$ & 22 & 20 & $30^{*}$ \\
$1: 2$ & 15 & 26 & 19 \\
$1: 4$ & 12 & 17 & 23 \\
$1: 1$ & 16 & 15 & 10
\end{tabular}

Table 4. The number of sessions conducted in each condition for each pigeon in Experiment 2.

Stability criteria were met in each condition by each pigeon excluding those indicated with *. 


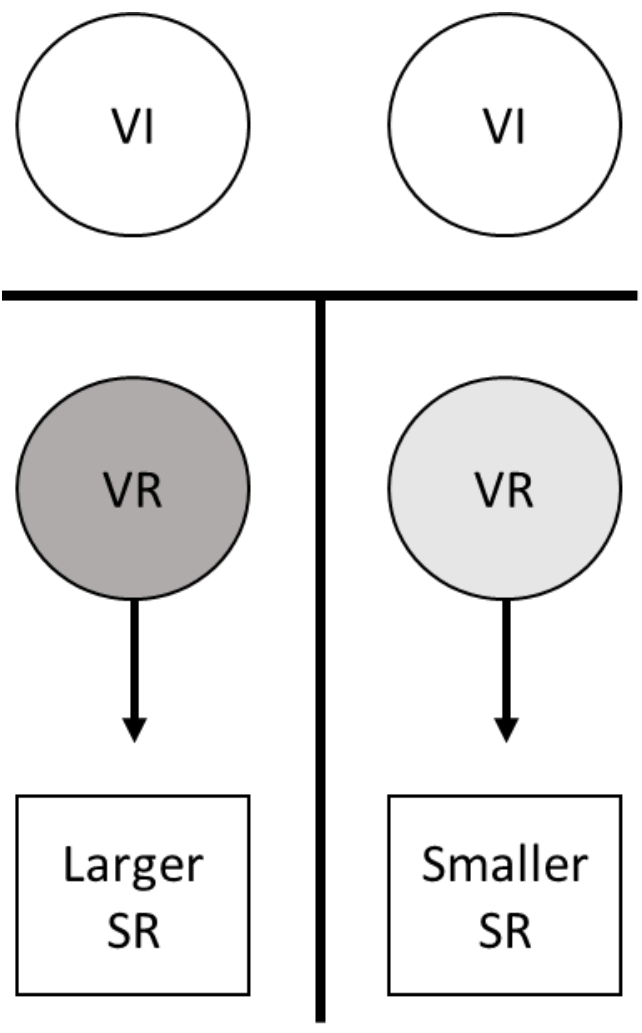

Figure 1. Free-choice trial operation of the concurrent-chain schedule in Experiment 1 with the concurrently available VI schedules of the initial link shown at the top, the VR schedules of the terminal links shown in the middle, and the reinforcers shown on the bottom. Note: the larger SR varied between 2-, 4-, and 8-s depending on the condition of the experiment and the smaller SR remained constant at 2-s throughout the experiment. 

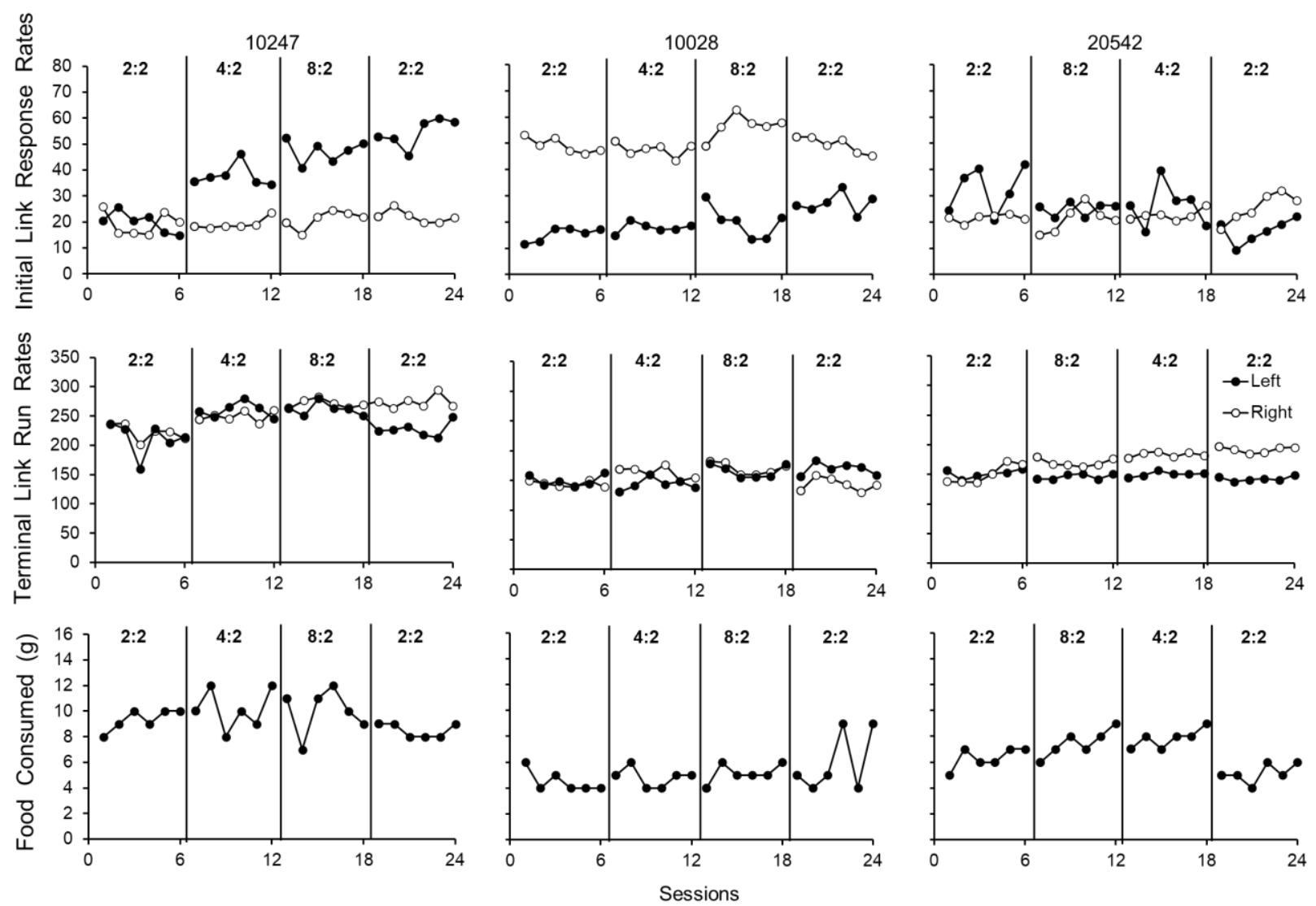

Figure 2. In Experiment 1, free-choice trial initial-link response rates are shown in responses per minute (top row) and terminal-link run rates shown in responses per minute excluding PRP time (middle row). Food consumed per session (bottom row) are shown for each pigeon (columns) as well. 

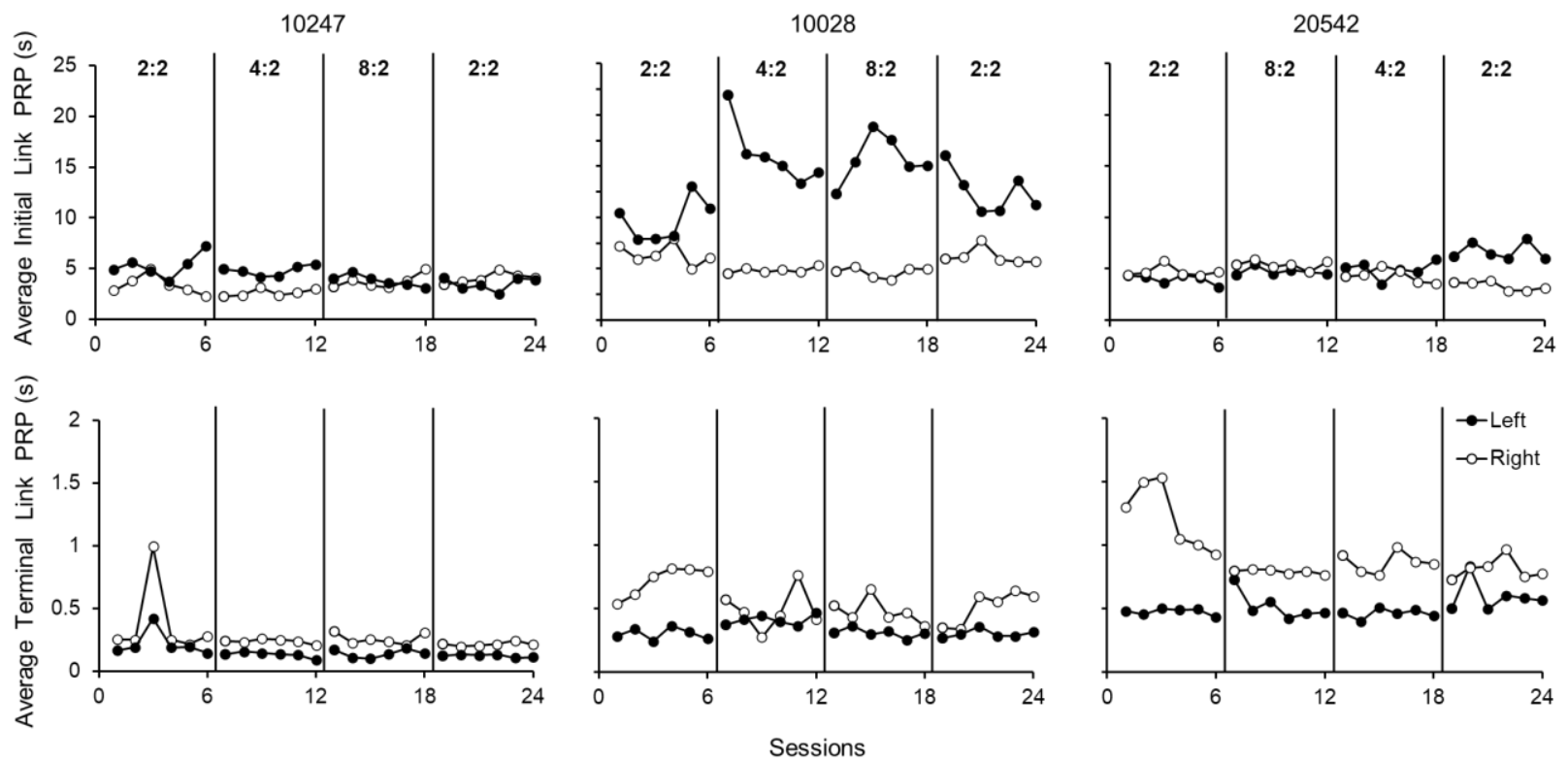

Figure 3. In Experiment 1, free-choice trial average initial-link PRP (top row) and average terminal-link PRP (bottom row) are shown for each pigeon (columns).

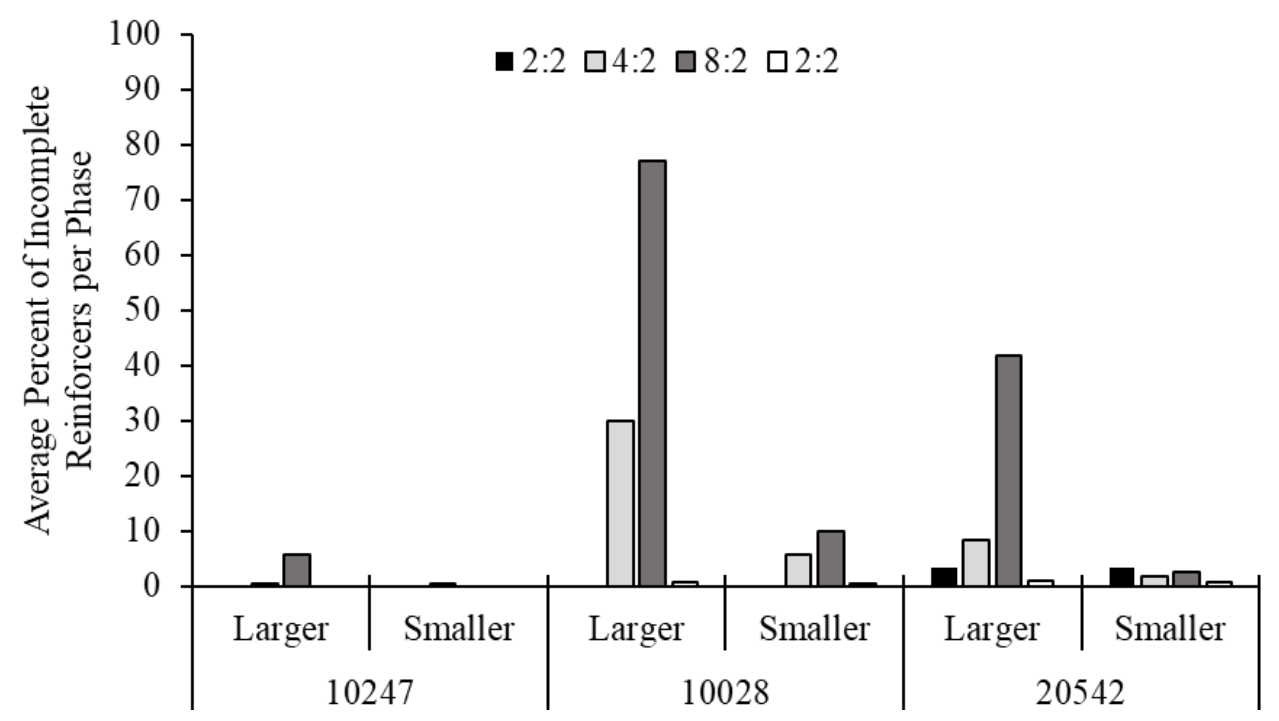

Figure 4. In Experiment 1, average percent of reinforcers that ended due to the limited hold in each phase for each pigeon on the larger and smaller chains. 

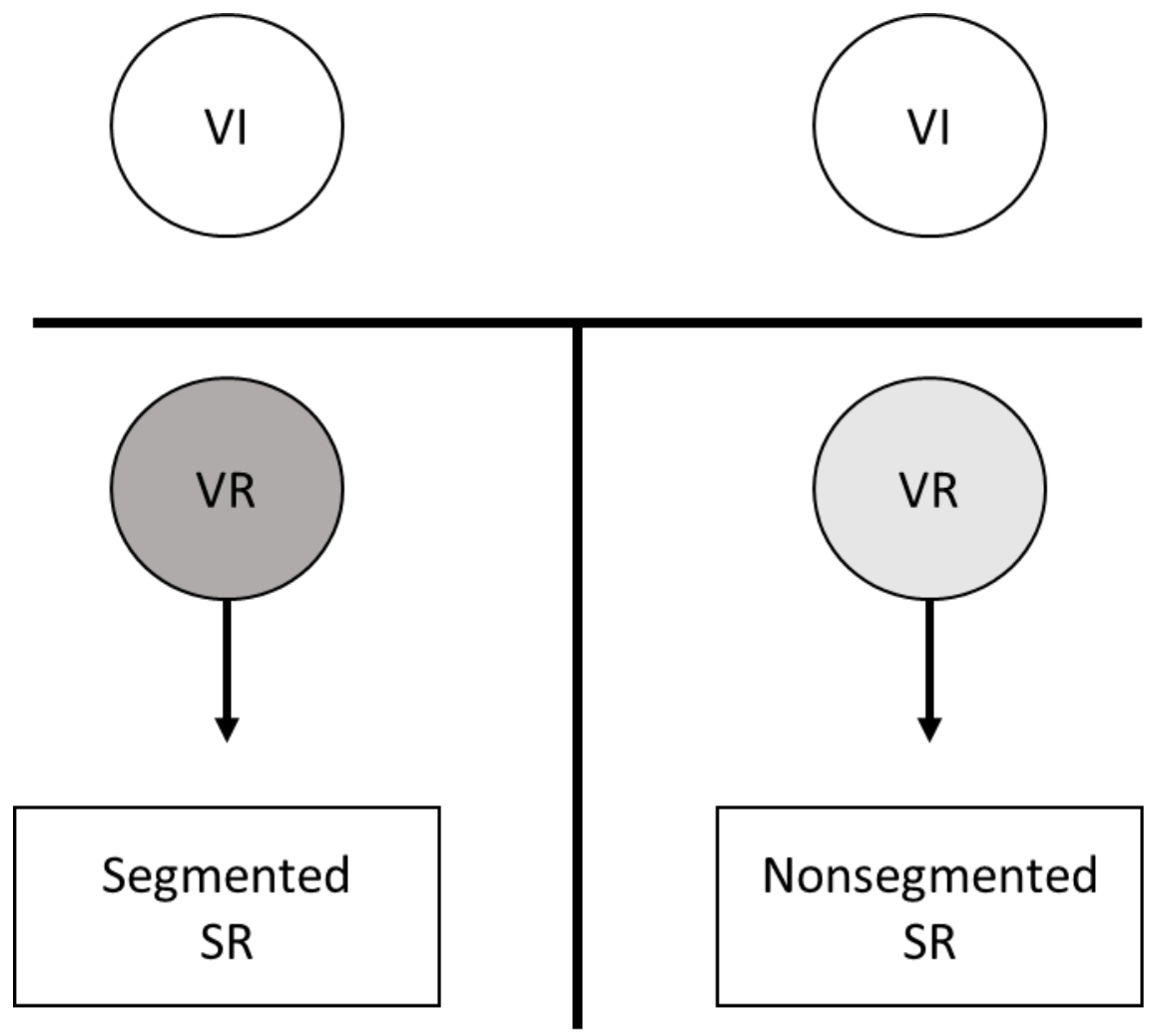

Figure 5. Free-choice trial operation of the concurrent-chain schedule in Experiment 2 with the concurrently available VI schedules of the initial link shown at the top, the VR schedules of the terminal links shown in the middle, and the reinforcers shown on the bottom. Note: the segmented SR consisted of one 8-, two 4-, or four 2-s access periods per IRI depending on the condition of the experiment and the nonsegmented SR remained constant at one 8-s access period throughout the experiment. 

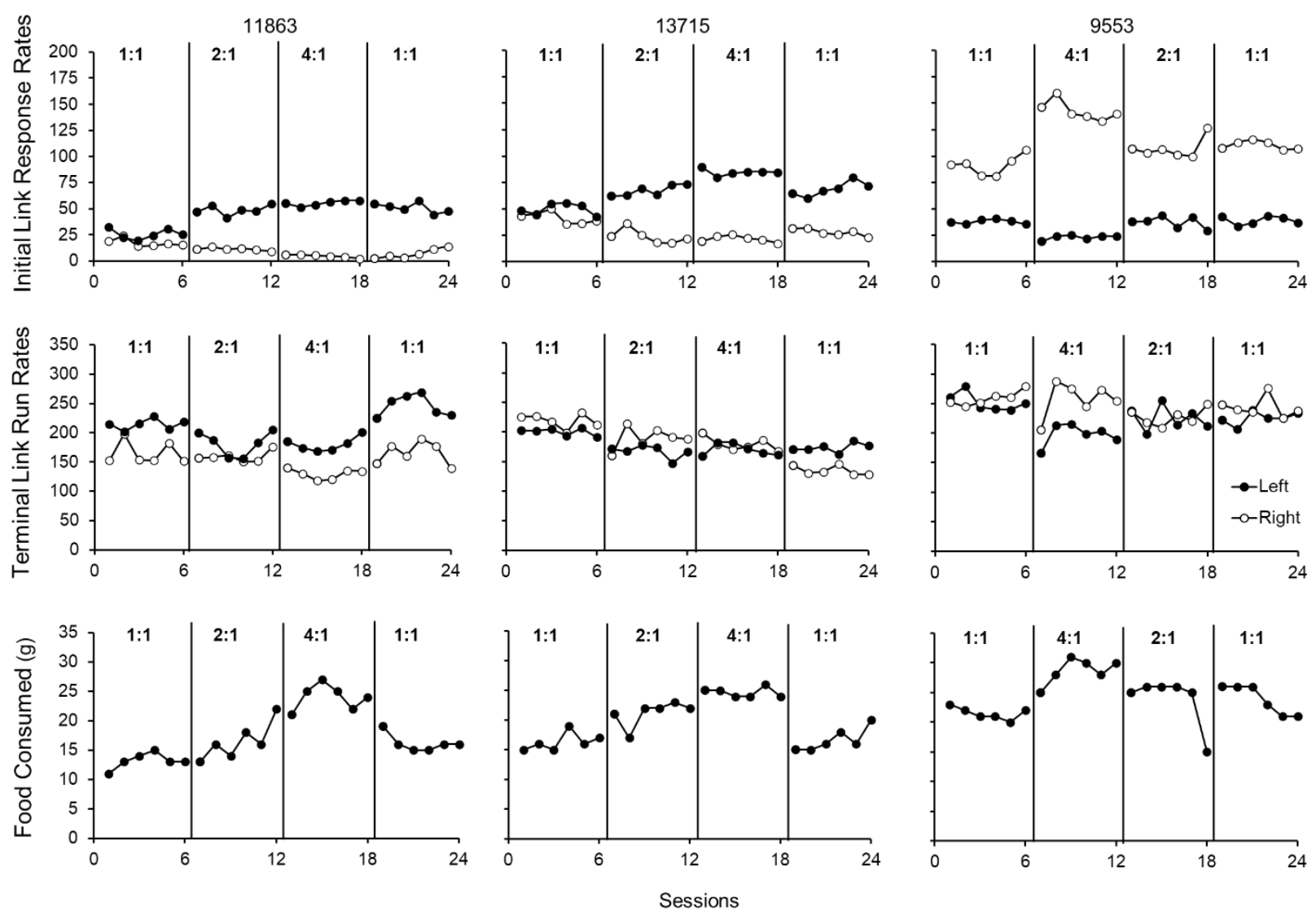

Figure 6. In Experiment 2, free-choice trial initial-link response rates are shown in responses per minute (top row) and terminal-link run rates shown in responses per minute excluding PRP time (middle row). Food consumed per session (bottom row) are shown for each pigeon (columns) as well. 

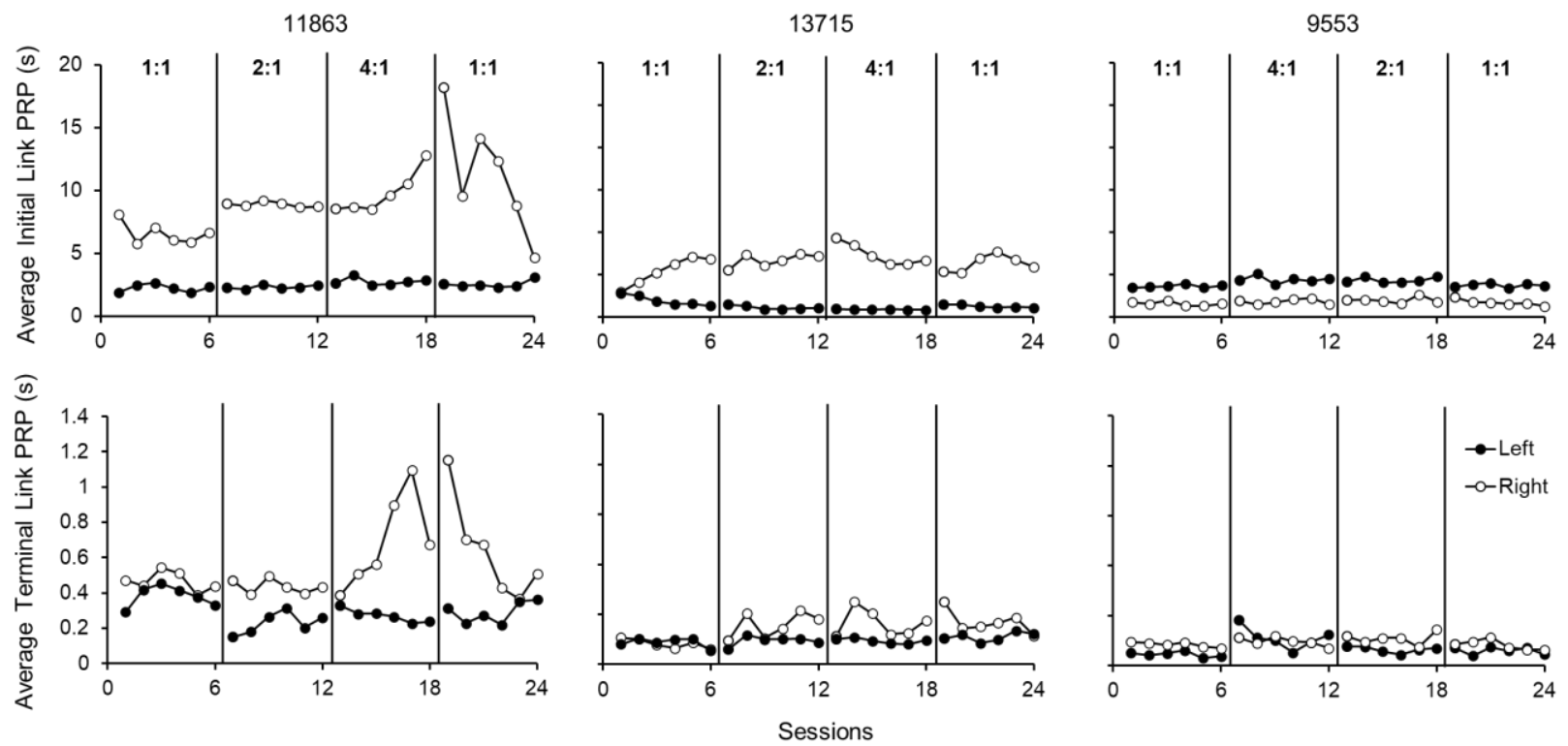

Figure 7. In Experiment 2, free-choice trial average initial-link PRP (top row) and average terminal-link PRP (bottom row) are shown for each pigeon (columns).
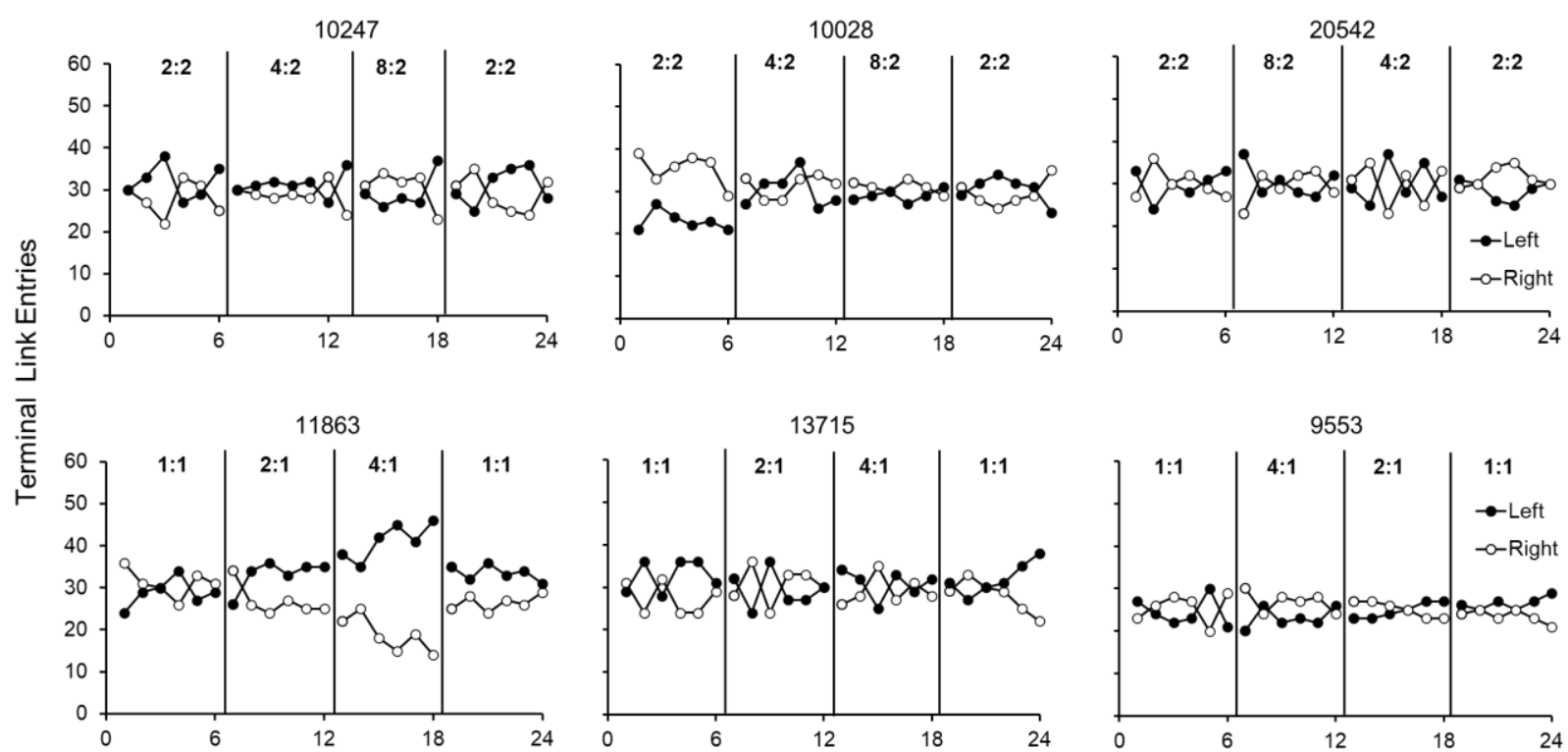

Figure 8. In Experiment 1 (top) and Experiment 2 (bottom), terminal link entries are shown per session. 

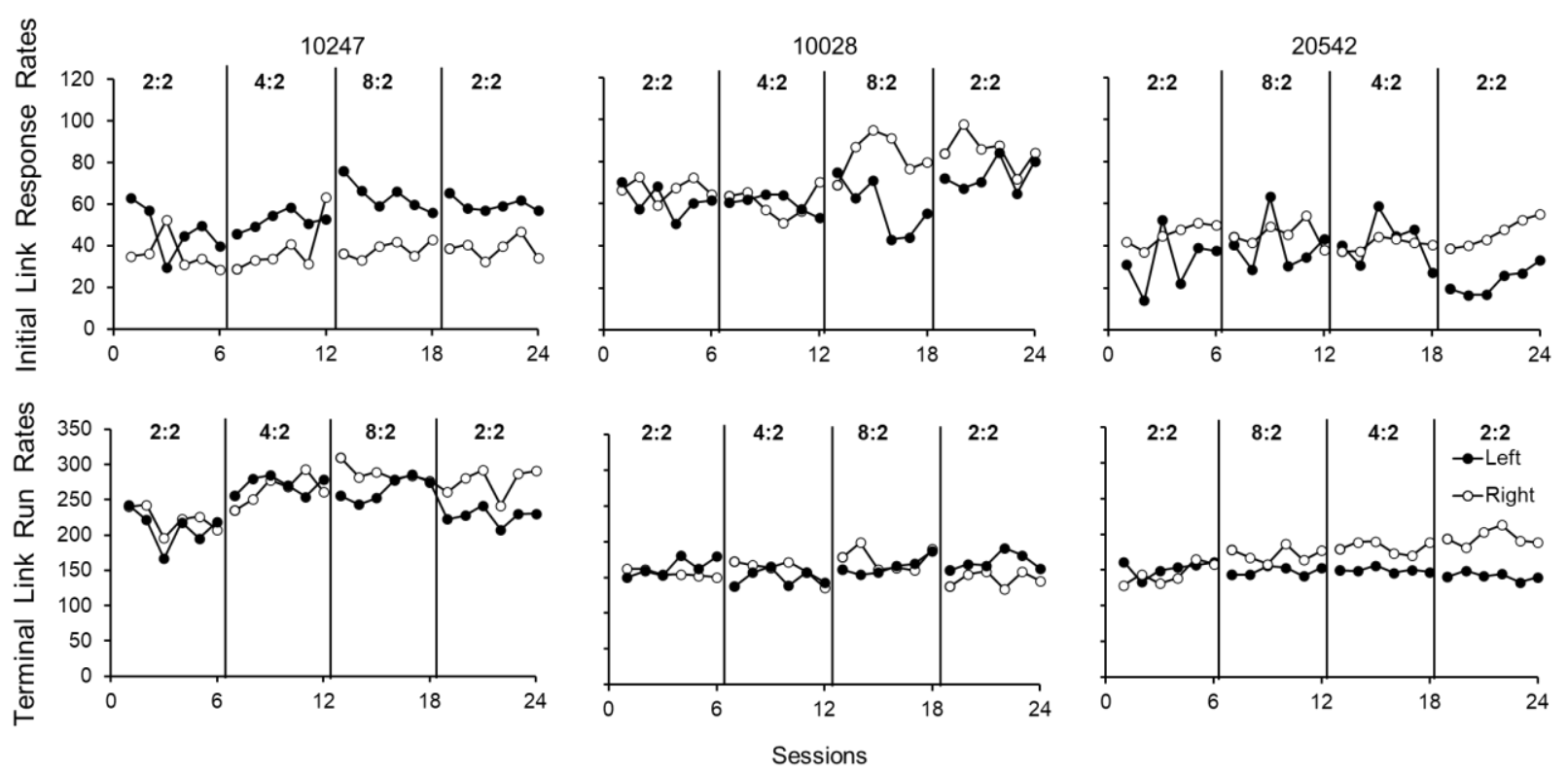

Figure 9. In Experiment 1, forced-choice trial initial-link response rates are shown in responses per minute (top row) and terminal-link run rates shown in responses per minute excluding PRP time (bottom row) for each pigeon (columns). 

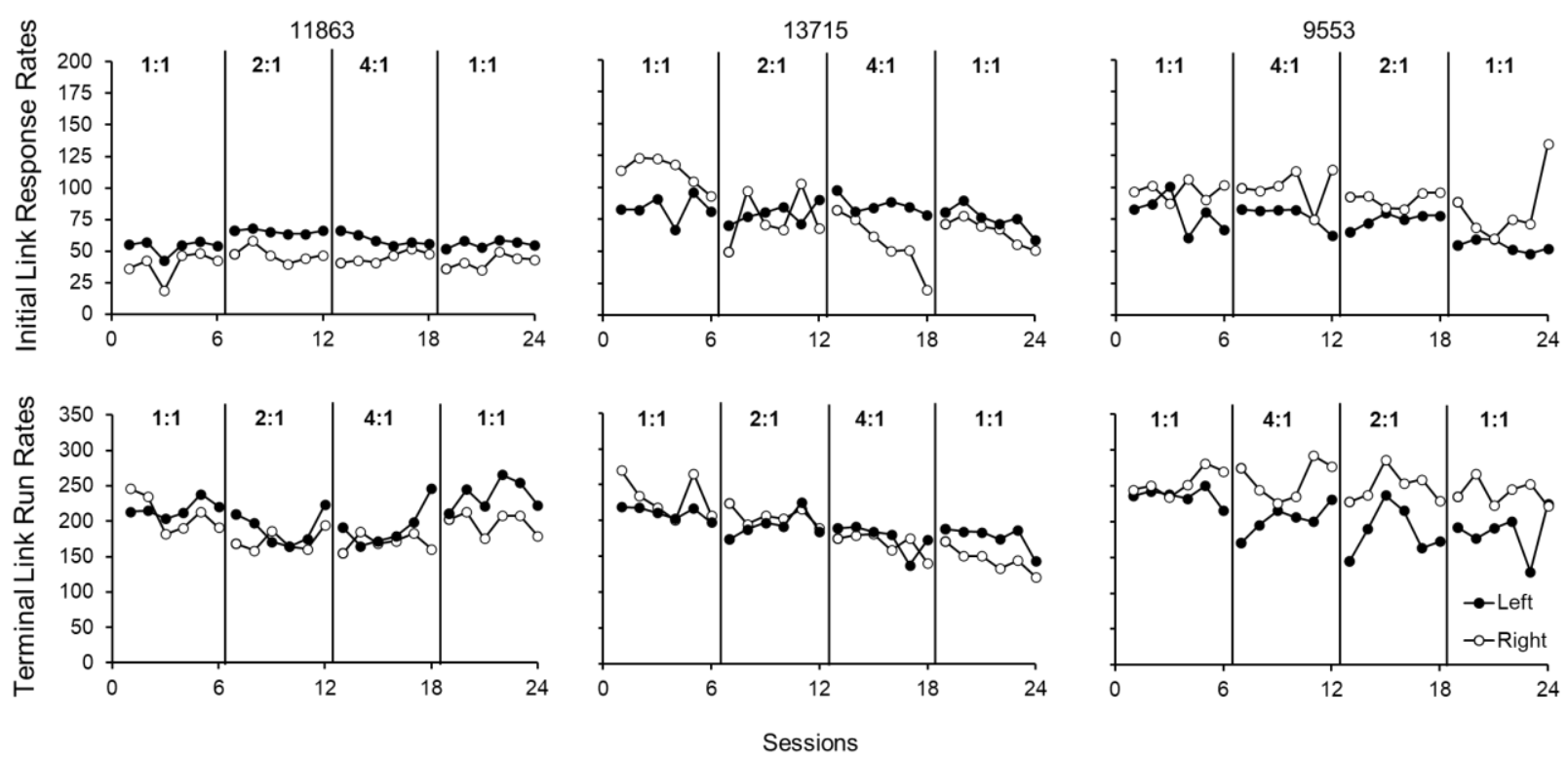

Figure 10. In Experiment 2, forced-choice trial initial-link response rates are shown in responses per minute (top row) and terminal-link run rates shown in responses per minute excluding PRP time (bottom row) for each pigeon (columns). 